\title{
Coherent flavour oscillation and CP violating parameter in thermal resonant leptogenesis
}

\author{
Satoshi Iso and Kengo Shimada \\ High Energy Accelerator Research Organization (KEK), \\ The Graduate University for Advanced Studies (SOKENDAI), \\ Oho 1-1, Tsukuba, Ibaraki 305-0801, Japan \\ E-mail: satoshi.iso@kek.jp, skengo@post.kek.jp
}

ABStract: Solving the Kadanoff-Baym (KB) equations in a different method from our previous analysis, we obtain the $\mathrm{CP}$ violating parameter $\varepsilon$ in the thermal resonant leptogenesis without assuming smallness of the off-diagonal Yukawa couplings. For that purpose, we first derive a kinetic equation for density matrix of $\mathrm{RH}$ neutrinos with almost degenerate masses $M_{i}(i=1,2) \sim M$. If the deviation from thermal equilibrium is small, the differential equation is reduced to a linear algebraic equation and the density matrix can be solved explicitly in terms of the time variation of (local) equilibrium distribution function. The obtained $\mathrm{CP}$-violating parameter $\varepsilon_{i}$ is proportional to an enhancement factor $\left(M_{i}^{2}-M_{j}^{2}\right) M_{i} \Gamma_{j} /\left(\left(M_{i}^{2}-M_{j}^{2}\right)^{2}+R_{i j}^{2}\right)$ with a regulator $R_{i j}=M\left(\Gamma_{i}+\Gamma_{j}\right)$, consistent with the previous analysis. The decay width can be determined systematically by the 1PI self-energy of the RH neutrinos in the 2PI formalism.

Keywords: Neutrino Physics, Thermal Field Theory, Cosmology of Theories beyond the $\mathrm{SM}, \mathrm{CP}$ violation

ARXIV EPRINT: 1404.4816 


\section{Contents}

1 Introduction 1

2 Comparison of time scales 3

3 From KB to density matrix evolution 4

3.1 Green functions 5

3.2 Kadanoff-Baym equations 5

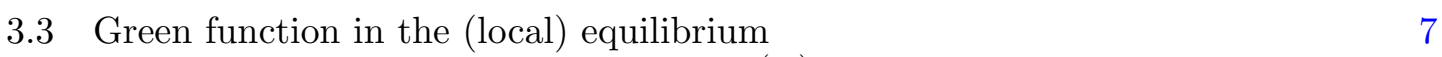

$\begin{array}{lll}3.4 & \mathrm{~KB} \text { equation for small deviation from } G_{\lessgtr}^{(\text {eq })} & 7\end{array}$

4 Kinetic equation for density matrix $\quad 10$

$\begin{array}{ll}4.1 \text { Kinetic equation for RH neutrinos } & 10\end{array}$

$\begin{array}{lll}4.2 & \text { Kinetic equation for lepton number } & 13\end{array}$

$\begin{array}{lll}4.3 & \text { Kinetic equations in terms of Yield variables } & 14\end{array}$

5 Solution of the kinetic equations $\quad \mathbf{1 6}$

$\begin{array}{lll}5.1 & \text { Formal solution of } \delta Y_{N} & 16\end{array}$

$\begin{array}{lll}\text { 5.2 CP-violation parameter } \varepsilon & 17\end{array}$

$\begin{array}{ll}5.3 \text { Explicit forms of } \delta Y_{N} & 18\end{array}$

5.4 CP violating parameter $\varepsilon$ when $\widetilde{\mathcal{C}}=0 \quad 21$

$\begin{array}{lll}6 & \text { Summary } & 22\end{array}$

A Derivation of the kinetic term $d_{t} f_{N} \quad 22$

B Explicit forms of $\mathcal{C}^{-1}$ and $\widetilde{\mathcal{C}}^{-1} \quad 24$

\section{Introduction}

Leptogenesis is one of very attractive scenario to explain the baryon number asymmetry [2] (for review, see [3]), but if the Majorana masses of the right-handed ( $\mathrm{RH})$ neutrinos have a hierarchical structure, the lightest Majorana mass must be heavier than $10^{9} \mathrm{GeV}$ [4] in order to produce sufficient amount of lepton number asymmetry. The condition can be evaded when Majorana masses are almost degenerate, which is called the resonant leptogenesis [7-9].

In light of the $\mathrm{LHC}$ experiment $\mathrm{TeV}$ scale leptogenesis has attracted much attention [11]-[38]. Especially, when we try to solve the naturalness problem via the Coleman Weinberg mechanism in a $B-L$ sector $[39,40], \mathrm{U}(1)_{B-L}$ gauge symmetry must be spontaneously broken around the $\mathrm{TeV}$ scale [41] and masses of $\mathrm{RH}$ neutrinos are naturally at the same energy scale. The leptogenesis scale can be much lowered by considering neutrino flavour oscillation out-of-equilibrium, which is important in the $\nu$ MSM scenario [42-45]. 
Hence it is becoming more and more important to treat coherent flavour oscillation in a systematic way.

In a conventional approach based on the classical Boltzmann equation, the evolution of the phase space distribution functions of on-shell particles is described and the interactions between particles are taken into account through the collision terms that comprise the S-matrix elements calculated separately. So the conventional classical method is not valid when the quantum coherent oscillation becomes important such as the flavour oscillations or the resonant leptogenesis. Density matrix formalism [46, 47] is a multi-flavour generalization of the Boltzmann equation and has been applied to neutrino flavour oscillations [48-51]. Another formulation is to use the Kadanoff-Baym (KB) equation, which is derived from the Schwinger-Dyson equation on closed-time-path. The approach is very systematic but difficult to solve without introducing various approximations. It was first applied to the leptogenesis with a hierarchical structure of the Majorana mass [52], and intensively used in various papers [53]-[64].

$\mathrm{KB}$ equation was applied to the resonant leptogenesis and oscillatory behaviour of lepton asymmetry was discussed [65-67]. The quantum oscillations in the flavored leptogenesis are also discussed in [68-72].

In the resonant leptogenesis, CP-asymmetry in the decay of $\mathrm{RH}$ neutrinos is generated by an interference of the tree and the self-energy one-loop diagrams. The $C P$-violating parameter is given by

$$
\varepsilon_{i} \equiv \frac{\Gamma_{N_{i} \rightarrow \ell \phi}-\Gamma_{N_{i} \rightarrow \overline{\ell \phi}}}{\Gamma_{N_{i} \rightarrow \ell \phi}+\Gamma_{N_{i} \rightarrow \overline{\ell \phi}}}=\sum_{j(\neq i)} \frac{\Im\left(h^{\dagger} h\right)_{i j}^{2}}{\left(h^{\dagger} h\right)_{i i}\left(h^{\dagger} h\right)_{j j}} \frac{\left(M_{i}^{2}-M_{j}^{2}\right) M_{i} \Gamma_{j}}{\left(M_{i}^{2}-M_{j}^{2}\right)^{2}+R_{i j}^{2}}
$$

where $h$ is the neutrino Yukawa coupling and $\Gamma_{i} \simeq\left(h^{\dagger} h\right)_{i i} M_{i} / 8 \pi$ is the decay width of $N_{i}$. The resonant enhancement of the CP-violating parameter was discussed in [73], and systematically studied in $[8,74,75]$. The regulator was given by $R_{i j}=M_{i} \Gamma_{j}$. If the mass difference is larger than the decay width, we have $\left|M_{i}^{2}-M_{j}^{2}\right| \gg R_{i j}$, and $\varepsilon_{i}$ is suppressed by $\Gamma_{i} / M \sim\left(h^{\dagger} h\right)_{i i}$. However, in the degenerate case, $\left|M_{i}-M_{j}\right| \sim \Gamma$ and $\varepsilon$ can be enhanced to $\mathcal{O}\left(\left(h^{\dagger} h\right)^{0}\right) \sim 1$. Hence the determination of the regulator $R_{i j}$ is essential for a precise prediction of the lepton number asymmetry in the resonant leptogenesis. The authors [76] calculated the resummed propagator of the $\mathrm{RH}$ neutrinos and obtained a different regulator $R_{i j}=\left|M_{i} \Gamma_{i}-M_{j} \Gamma_{j}\right|$. By using their result, the enhancement factor becomes much larger. The origin of the difference of the regulators is discussed in [77, 78].

Recently Garny et al. [79] systematically investigated generation of the lepton asymmetry in the resonant leptogenesis using the formulas developed in [53, 54]. They discussed the $\mathrm{CP}$ violating asymmetries generated in the decay of initial $\mathrm{RH}$ neutrinos and studied effects of particle mixing due to a small mass splitting [6] based on the non-equilibrium quantum field theory. In the investigation, they considered a non-equilibrium initial condition in a time-independent background and calculated generation of the lepton number asymmetry. Starting from the vacuum initial state for the RH neutrinos, they read the CP-violating parameter from the generated lepton number asymmetry. The effective regulator they derived is $R_{i j}=M_{i} \Gamma_{i}+M_{j} \Gamma_{j}$, which differs from the previous results. 
In a previous paper [1], we solved the $\mathrm{KB}$ equation in the thermal resonant leptogenesis and obtained the same regulator $R_{i j}=M_{i} \Gamma_{i}+M_{j} \Gamma_{j}$ as above. Our derivation is applicable to cases when the background is slowly changing with time but valid only when the offdiagonal component of the Yukawa couplings are small compared to the diagonal ones

$$
\Re\left(h^{\dagger} h\right)^{\prime}<\left|M_{i}-M_{j}\right| / M \simeq \Gamma / M \sim\left(h^{\dagger} h\right)_{i i}^{d} .
$$

For practical purposes, this condition is too strong and it is desirable to extend the analysis to more general cases with large off-diagonal Yukawa couplings.

The purpose of the paper is to solve the KB equation without assuming smallness of the off-diagonal Yukawa couplings (1.2). In order for it, we first rewrite the KB equation in terms of the density matrix of $\mathrm{RH}$ neutrinos. Since Majorana fermions have 2 spinor components, the density matrix is $2 N_{F} \times 2 N_{F}$ for $N_{F}$ flavours. In deriving the kinetic equation for the density matrix, we assume that deviation of the distribution functions are not very large. If the condition is satisfied, we reproduce the equation [46]. Various terms in the equation can be systematically obtained in the 2PI formalism. The kinetic equation, which is a differential equation, is reduced to a linear equation when an inequality $H \ll \Gamma_{i}$ in (2.6) between the Hubble parameter $H$ and the decay width $\Gamma_{i}$ of $\mathrm{RH}$ neutrino $N_{i}$ is satisfied. Then it is straightforward to obtain the solution of deviation of the RH neutrino density matrix from the local equilibrium. From the off-diagonal component of density matrix, we can read the $\mathrm{CP}$ violating parameter $\varepsilon$. The same $\mathrm{CP}$ violating parameter as in [1] with the regulator $R_{i j}=M_{i} \Gamma_{i}+M_{j} \Gamma_{j}$ is obtained.

The paper is organized as follows. In section 3, we derive kinetic equations of density matrices starting from the Kadanoff-Baym equations. The derivation is performed under an assumption that distribution functions are not far from the local equilibrium ones. But smallness of flavour mixing interactions is not assumed. Namely, the off-diagonal Yukawa couplings are not necessary small compared to the diagonal ones, and coherent flavour oscillation is fully taken into account. In section 4 , we derive kinetic equations of the RH neutrinos and lepton asymmetry in the yield variables. In section 5 , we solve the kinetic equations to obtain the $\mathrm{RH}$ neutrino density matrix. From the flavour offdiagonal component, we read the CP-violating parameter $\varepsilon$. We summarize in section 6 . In appendix A, we explain derivation of the kinetic term $d_{t} f$ from $\mathrm{KB}$ equation. Explicit forms of inverse of matrix $\mathcal{C}$ are written in appendix B.

\section{Comparison of time scales}

We introduce multi-flavour right-handed neutrinos $\nu_{R, i}$ where $i$ is the flavour index, $i=1 \cdots N_{F}$. In particular we consider a case that two $\mathrm{RH}$ neutrinos have almost degenerate masses. Hence we set $N_{F}=2$ in the following. We write $N_{i}=\nu_{R, i}+\nu_{R, i}^{c}$. The Lagrangian is given by

$$
\begin{aligned}
\mathcal{L} & =\mathcal{L}_{\mathrm{SM}}+\frac{1}{2} \bar{N}^{i}\left(i \not \nabla-M_{i}\right) N^{i}+\mathcal{L}_{\mathrm{int}} \\
\mathcal{L}_{\mathrm{int}} & \equiv-h_{\alpha i}\left(\bar{\ell}_{a}^{\alpha} \epsilon_{a b} \phi_{b}^{*}\right) P_{R} N^{i}+h_{i \alpha}^{\dagger} \bar{N}^{i} P_{L}\left(\phi_{b} \epsilon_{b a} \ell_{a}^{\alpha}\right)
\end{aligned}
$$


where $\alpha, \beta=1,2,3$ and $a, b=1,2$ are flavor indices of the SM leptons $\ell_{a}^{\alpha}$ and isospin $\mathrm{SU}(2)_{L}$ indices respectively. $M_{i}$ is the Majorana mass of $N_{i}$ and $h_{i \alpha}$ is the Yukawa coupling of $N^{i}, \ell_{a}^{\alpha}$ and the Higgs $\phi_{a}$ doublet. $P_{R(L)}$ are chiral projections on right(left)-handed fermions. As a concrete model we consider the Lagrangian (2.2) with only the Yukawa couplings, but the following analysis and the results are not restricted to the specific model: we can systematically include other interactions such as the $B-L$ gauge interactions of the $\mathrm{RH}$ neutrinos $N_{i}$.

We compare various time (or inverse mass) scales in the model. First the Hubble parameter $H$ in the radiation dominant universe is given by

$$
H \sim 1.66 \sqrt{g_{*}} \frac{T^{2}}{M_{p l}} \sim \frac{T^{2}}{10^{18} \mathrm{GeV}}
$$

where $T$ is the temperature of the universe. Thermal masses and decay widths of SM leptons $\ell$ and Higgs $\phi$ are given by $m_{\ell, \phi} \sim g T$ and $\Gamma_{\ell, \phi} \sim g^{2} T$ where $g$ is the SM gauge coupling. When $T$ is lower than $g^{2} \times 10^{18} \mathrm{GeV}, \Gamma_{\ell, \phi}$ are larger than $H$. Since we are interested in the $\mathrm{TeV}$ scale leptogenesis in the present paper, we have the relation

$$
\Gamma_{\ell, \phi} \sim g^{2} T \gg H \sim \frac{T^{2}}{10^{18} \mathrm{GeV}} .
$$

In type I seasaw model, the decay width of the RH neutrino is given by $\Gamma_{i} \sim\left(h^{\dagger} h\right)_{i i} M_{i} / 8 \pi$. The ratio of $\Gamma_{i}$ to the Hubble parameter (2.3) at temperature $T=M_{i}$ is rewritten in terms of the "effective neutrino mass" $\tilde{m}_{i}$ as (see e.g. [3])

$$
K_{i}=\frac{\Gamma_{i}}{H\left(M_{i}\right)}=\frac{\tilde{m}_{i}}{10^{-3} \mathrm{eV}}, \quad \tilde{m}_{i} \equiv \frac{\left(h^{\dagger} h\right)_{i i} v^{2}}{M_{i}},
$$

where $v$ is the scale of the EWSB. Hence if we take the Yukawa coupling so as to $\tilde{m}_{i} \sim$ $0.1 \mathrm{eV}$, the ratio becomes $K_{i} \sim 100$. This corresponds to the strong washout regime. Hence we have the following inequality among various quantities with mass dimension:

$$
\Gamma_{\phi}, \Gamma_{\ell} \gg \Gamma_{i} \gg H
$$

The inequality $\Gamma_{\ell, \phi} \gg \Gamma_{i}$ is not used in the analysis of the present paper. Hence our results are still valid when the RH neutrinos are charged under $B-L$ gauge interaction and $\Gamma_{i}$ becomes larger.

\section{From KB to density matrix evolution}

In this section, we derive an evolution equation of the multi-flavour density matrix of the $\mathrm{RH}$ neutrinos $N_{i}[46,50]$ starting from the Kadanoff-Baym equation. We extend the method established in the flat space-time by [67] to the case of the expanding universe. In [67], the evolution equation in the expanding universe was derived by replacing the physical time and the Majorana mass $M$ by the conformal time and $a M$, where $a$ is the scale factor. Our result in the following agrees with the result in [67], and give a justification of their method to obtain the evolution equation in the expanding universe. KB equation is derived 
from the Schwinger-Dyson equation on the closed-time-path, which is a fully systematic equation of the Green functions in a non-equilibrium setting. Deriving the kinetic equation for density matrix from the KB equation makes it clear under what conditions the density matrix equation is obtained and what kinds of diagrams contribute to various terms in the density matrix formalism, especially the resonantly enhanced CP violating parameter and the decay widths $\Gamma_{i}$ contained in the regulator of $\varepsilon_{i}$.

\subsection{Green functions}

First we define various Green functions. An $i j$-component of Wightman Green functions is defined by

$$
G_{>}(x, y)_{i j}=\left\langle\hat{N}_{i}(x) \overline{\hat{N}_{j}}(y)\right\rangle, \quad G_{<}(x, y)_{i j}=-\left\langle\overline{\hat{N}_{j}}(y) \hat{N}_{i}(x)\right\rangle .
$$

The mass $\hat{M}$ and 1PI self-energy function $\Pi$ are also $2 \times 2$ matrices (besides the spinor structure) with the flavour indices $i j$. We also define the spectral function by $G_{\rho}=i\left(G_{>}-G_{<}\right)$ and the statistical propagator by $G_{F}=\left(G_{>}+G_{<}\right) / 2$. The retarded (advanced) Green functions are related to the spectral function by the relation

$$
G_{R / A}(x, y)= \pm \Theta\left( \pm\left(x^{0}-y^{0}\right)\right) G_{\rho}(x, y) .
$$

For the self-energy function $\Gamma$, we can similarly define various types of self-energy functions of $R, A, \rho$ and $\gtrless$. (See appendix $\mathrm{B}$ of [1].)

\subsection{Kadanoff-Baym equations}

The Kadanoff-Baym (KB) equation of the $\mathrm{RH}$ neutrinos in the expanding universe is given by

$$
\left(i \gamma^{0} \partial_{x^{0}}-\frac{\mathbf{q} \cdot \boldsymbol{\gamma}}{a\left(x^{0}\right)}-\hat{M}\right) G_{\lessgtr}\left(x^{0}, y^{0}\right)-\left(\Pi_{R} * G_{\lessgtr}\right)\left(x^{0}, y^{0}\right)=\left(\Pi_{\lessgtr} * G_{A}\right)\left(x^{0}, y^{0}\right) .
$$

$\mathbf{q}$ is the comoving momentum and $*$ is the convolution in the time coordinate. Symbolically we write it as

$$
i G_{0}^{-1} G_{\lessgtr}-\Pi_{R} G_{\lessgtr}=\Pi_{\lessgtr} G_{A} .
$$

The 1PI self-energy function $\Pi$ of $\mathrm{RH}$ neutrino is obtained by cutting a (full) propagator of 2PI diagrams. In the 2PI formalism, all internal lines represent full propagators while vertices are tree. For more details, see appendix C, D of [1]. Figure 1 are examples of self-energy diagrams. In deriving the $\mathrm{KB}$ equation, figure 1 (a) gives the decay width at tree level while figure 1(b) gives an interference between the tree and the one-loop vertex diagrams [63]. Hence the direct CP violating parameter is contained in figure 1(b). If we include $Z^{\prime}$ gauge boson or a scalar field coupled with the RH neutrinos, other self-energy diagrams in figure 2 contribute to $\Pi$.

By taking the Fourier transform with respect to the relative time coordinate $s=x^{0}-y^{0}$, eq. (3.3) becomes

$$
e^{-i \diamond}\left\{\gamma^{0} q_{0}-\frac{\mathbf{q} \cdot \gamma}{a(X)}-\hat{M}-\Pi_{R}\left(X ; q_{0}\right)\right\}\left\{G_{\lessgtr}\left(X ; q_{0}\right)\right\}=e^{-i \diamond}\left\{\Pi_{\lessgtr}\left(X ; q_{0}\right)\right\}\left\{G_{A}\left(X ; q_{0}\right)\right\} .
$$




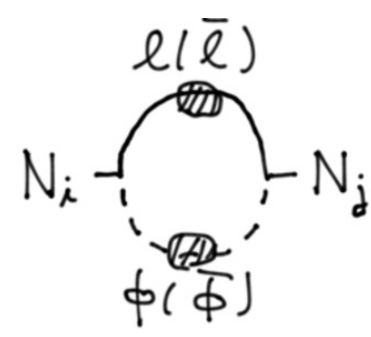

(a)

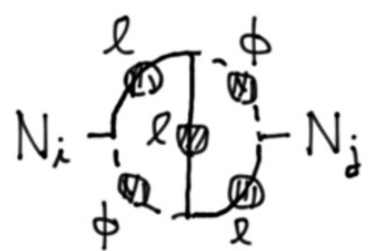

(b)

Figure 1. Self-energy diagrams of $\mathrm{RH}$ neutrino $N_{i}$. In the 2PI formalism, each internal line represents a full propagator while vertices are given by tree vertices. Tree-level decay width is generated from the left figure (a). The right figure (b) gives the so-called direct $\mathrm{CP}$ violating parameter of the RH neutrino, an interference between the tree and the one-loop vertex corrections.

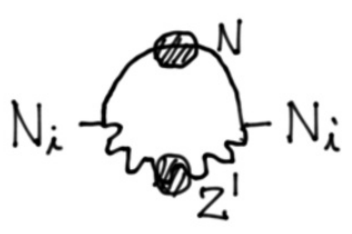

(a)

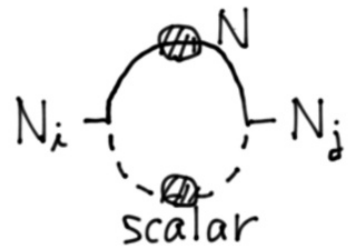

(b)

Figure 2. Self-energy diagrams of RH neutrino $N_{i}$ with $B-L$ gauge interaction (a) or with Majorana Yukawa interaction with a SM singlet scalar field (b).

$X=\left(x^{0}+y^{0}\right) / 2$ is the center-of-mass time coordinate. Here we used the Moyal-Weyl bracket defined by

$$
e^{-i \diamond}\left\{f\left(X ; q_{0}\right)\right\}\left\{g\left(X ; q_{0}\right)\right\}=e^{\frac{i}{2}\left(\partial_{q_{0}}^{f} \partial_{X}^{g}-\partial_{X}^{f} \partial_{q_{0}}^{g}\right)} f\left(X ; q_{0}\right) g\left(X ; q_{0}\right) .
$$

In the expanding universe with the Hubble parameter $H, X$ derivative is often estimated as $\partial_{X} \sim \mathcal{O}(H)$. On the other hand, derivative with respect to the relative momentum $q_{0}$ is estimated as $\partial_{q_{0}} f \sim \mathcal{O}\left(1 / \Gamma_{f}\right)$ where $\Gamma_{f}$ is the decay width of the function $f(X, s) \sim$ $e^{-\Gamma_{f} s}$. In (3.5), $\Gamma$ for $G_{*}(*=\lessgtr, A, R,,$,$) is given by the decay width \Gamma_{N}$ of the $\mathrm{RH}$ neutrinos. In the strong washout regime, we have an inequality $H \ll \Gamma_{N}$. Since the dominant contribution to the self-energy $\Pi$ comes from the diagram in figure $1(\mathrm{a}), \Gamma$ for $\Pi_{*}$ is given by the decay widths of the charged lepton and Higgs $\Gamma_{l, \phi}$ propagating in the internal lines. They are much larger than $\Gamma_{N}$. An expansion with respect to $\diamond$ is given by $H / \Gamma_{N, \ell, \phi}$ and hence justified by (2.6).

Taking up to the first order of the derivative expansion of $\diamond$, we have

$$
\begin{aligned}
& \left(\gamma^{0} q_{0}-\frac{\mathbf{q} \cdot \gamma}{a}-\hat{M}-\Pi_{R}\right) G_{\lessgtr}-i \diamond\left\{\gamma^{0} q_{0}-\frac{\mathbf{q} \cdot \gamma}{a}-\hat{M}-\Pi_{R}\right\}\left\{G_{\lessgtr}\right\} \\
& \quad=\Pi_{\lessgtr} G_{A}-i \diamond\left\{\Pi_{\lessgtr}\right\}\left\{G_{A}\right\} .
\end{aligned}
$$

The spectral function $G_{\rho}$ satisfies a similar equation in which $\gtrless($ of $G$ and $\Pi)$ is replaced by $\rho$. 


\subsection{Green function in the (local) equilibrium}

If we drop the derivative term containing $\diamond$, it becomes an equation for the Green function in the local equilibrium at time $X$;

$$
\left(\gamma^{0} q_{0}-\frac{\mathbf{q} \cdot \gamma}{a}-\hat{M}-\Pi_{R}^{\mathrm{eq}}\right) G_{\lessgtr}^{\mathrm{eq}}=\Pi_{\lessgtr}^{\mathrm{eq}} G_{A}^{\mathrm{eq}} .
$$

By using the KB equation of the retarded Green fucntion (see eq. (2.11) in [1]),

$$
\left(\gamma^{0} q_{0}-\frac{\mathbf{q} \cdot \gamma}{a}-\hat{M}-\Pi_{R}^{\mathrm{eq}}\right) G_{R}^{\mathrm{eq}}=-1,
$$

eq. (3.8) is solved as

$$
G_{\lessgtr}^{\mathrm{eq}}=-G_{R}^{\mathrm{eq}} \Pi_{\lessgtr}^{\mathrm{eq}} G_{A}^{\mathrm{eq}} .
$$

In the thermal equilibrium at temperature $T$, the Green functions are anti-periodic in the time direction with an imaginary period $i \beta=i / T$. Hence Fourier transform satisfies the Kubo-Martin-Schwinger (KMS) relation

$$
G_{\gtrless}^{(\mathrm{eq})}(q)=-i\left\{\begin{array}{c}
1-f^{(\mathrm{eq})}(q) \\
-f^{(\mathrm{eq})}(q)
\end{array}\right\} G_{\rho}^{(\mathrm{eq})}(q),
$$

where $f^{(\mathrm{eq})}$ is the Fermi distribution function $f^{(\mathrm{eq})}\left(q_{0}\right)=1 /\left(e^{q_{0} / T}+1\right)$. Various properties of the equilibrium Green functions are reviewed in section 3.5 in [1]. Especially, as shown in (3.45) in [1], the off-diagonal component of the Wightman functions $G_{\gtrless}^{\prime(\mathrm{eq})}\left(x_{0}, y_{0}\right)$ vanishes in the limit of $x_{0} \rightarrow y_{0}$. It directly follows from the KMS relation together with the equaltime anti-commutation relation of the fields $N_{i}$. When the system is out of equilibrium, it deviates from zero whose imaginary part gives the $\mathrm{CP}$ violating source for the lepton number asymmetry.

If the system is slightly deviated from the local equilibrium, KMS relation indicates that the deviation is written as

$$
\delta G_{\gtrless}(q)=-i \delta\left\{\begin{array}{c}
1-f(q) \\
-f(q)
\end{array}\right\} G_{\rho}(q)-i\left\{\begin{array}{c}
1-f(q) \\
-f(q)
\end{array}\right\} \delta G_{\rho}(q) .
$$

We then define

$$
\widetilde{\delta G}_{\lessgtr} \equiv \delta G_{\lessgtr}+i\left[\begin{array}{c}
-f \\
1-f
\end{array}\right] \delta G_{\rho}=\delta G_{F}+i\left(\frac{1}{2}-f\right) \delta G_{\rho},
$$

which represents a deviation of the distribution function $\widetilde{\delta G}_{\lessgtr} \sim i(\delta f) G_{\rho}$.

\subsection{KB equation for small deviation from $G_{\lessgtr}^{(\text {eq })}$}

We now derive the KB equation for a small deviation from the local equilibrium. Taking a variation in (3.7) and picking up to the first order terms of $\delta$, we have

$$
\begin{aligned}
& \left(\gamma^{0} q_{0}-\frac{\mathbf{q} \cdot \gamma}{a}-\hat{M}-\Pi_{R}^{\mathrm{eq}}\right) \delta G_{\lessgtr}-\delta \Pi_{R} G_{\lessgtr}^{\mathrm{eq}}-i \diamond\left\{\gamma^{0} q_{0}-\frac{\mathbf{q} \cdot \gamma}{a}-\hat{M}-\Pi_{R}^{\mathrm{eq}}\right\}\left\{\delta G_{\lessgtr}\right\} \\
& -i \diamond\left\{\gamma^{0} q_{0}-\frac{\mathbf{q} \cdot \gamma}{a}-\hat{M}-\Pi_{R}^{\mathrm{eq}}-\delta \Pi_{R}\right\}\left\{G_{\lessgtr}^{\mathrm{eq}}\right\} \\
& \quad=\Pi_{\lessgtr}^{\mathrm{eq}} \delta G_{A}+\delta \Pi_{\lessgtr} G_{A}^{\mathrm{eq}}-i \diamond\left\{\Pi_{\lessgtr}^{\mathrm{eq}}\right\}\left\{G_{A}^{\mathrm{eq}}+\delta G_{A}\right\}-i \diamond\left\{\delta \Pi_{\lessgtr}\right\}\left\{G_{A}^{\mathrm{eq}}\right\} .
\end{aligned}
$$


We can obtain the same equation for $G_{\rho}$ by replacing $\lessgtr$ by $\rho$. By combining these equations and using the KMS relation, some terms are cancelled and we have

$$
\begin{aligned}
& \left(\gamma^{0} q_{0}-\frac{\mathbf{q} \cdot \gamma}{a}-\hat{M}-\Pi_{R}^{\mathrm{eq}}\right)\left(\delta G_{\lessgtr}+i\left[\begin{array}{c}
1-f \\
-f
\end{array}\right] \delta G_{\rho}\right) \\
& -i \diamond\left\{\gamma^{0} q_{0}-\frac{\mathbf{q} \cdot \boldsymbol{\gamma}}{a}-\hat{M}-\Pi_{R}^{\mathrm{eq}}\right\}\left(\left\{\delta G_{\lessgtr}\right\}+i\left[\begin{array}{c}
1-f \\
-f
\end{array}\right]\left\{\delta G_{\rho}\right\}\right) \\
& -i \diamond\left\{\gamma^{0} q_{0}-\frac{\mathbf{q} \cdot \boldsymbol{\gamma}}{a}-\hat{M}-\Pi_{R}^{\mathrm{eq}}-\delta \Pi_{R}\right\}\left\{\left[\begin{array}{c}
1-f \\
-f
\end{array}\right]\right\} G_{\rho}^{\mathrm{eq}}(-i) \\
& =\left(\delta \Pi_{\lessgtr}+i\left[\begin{array}{c}
1-f \\
-f
\end{array}\right] \delta \Pi_{\rho}\right) G_{A}^{\mathrm{eq}}-i(-i) \Pi_{\rho}^{\mathrm{eq}} \diamond\left\{\left[\begin{array}{c}
1-f \\
-f
\end{array}\right]\right\}\left\{G_{A}^{\mathrm{eq}}+\delta G_{A}\right\} \\
& \quad-i \diamond\left(\left\{\delta \Pi_{\lessgtr}\right\}+i\left[\begin{array}{c}
1-f \\
-f
\end{array}\right]\left\{\delta \Pi_{\rho}\right\}\right)\left\{G_{A}^{\mathrm{eq}}\right\}
\end{aligned}
$$

where we defined

$$
\left.\widetilde{\left\{\delta G_{\lessgtr}\right.}\right\} \equiv\left\{\delta G_{\lessgtr}\right\}+i\left[\begin{array}{c}
-f \\
1-f
\end{array}\right]\left\{\delta G_{\rho}\right\}=\left\{\delta G_{F}\right\}+i\left(\frac{1}{2}-f\right)\left\{\delta G_{\rho}\right\} .
$$

The deviation from $G_{\lessgtr}^{(\text {eq })}$ occurs due to the expansion of the universe, and hence $\delta G_{\lessgtr}$ is proportional to the Hubble parameter $H$. Since the derivative expansion of $\diamond$ is an expansion of $H$, we can drop terms containing more than one $\delta$ or $\diamond$ when $H \ll \Gamma_{N}, \Gamma_{\ell, \phi}$. Then (3.15) is simplified as

$$
\begin{aligned}
-i \diamond & \left\{\gamma^{0} q_{0}-\frac{\mathbf{q} \cdot \gamma}{a}-\hat{M}-\Pi_{R}^{\mathrm{eq}}\right\}\{i f\} G_{\rho}^{\mathrm{eq}}+i \Pi_{\rho}^{\mathrm{eq}} \diamond\{i f\}\left\{G_{A}^{\mathrm{eq}}\right\} \\
& =\widetilde{\delta \Pi_{\lessgtr}} G_{A}^{\mathrm{eq}}-\left(\gamma^{0} q_{0}-\frac{\mathbf{q} \cdot \gamma}{a}-\hat{M}-\Pi_{R}^{\mathrm{eq}}\right) \widetilde{\delta G_{\lessgtr}} .
\end{aligned}
$$

Instead of (3.4), we can start from

$$
i G_{\lessgtr} G_{0}^{-1}-G_{\lessgtr} \Pi_{A}=G_{R} \Pi_{\lessgtr}
$$

and obtain a similar equation to (3.16),

$$
\begin{gathered}
-i G_{\rho}^{\mathrm{eq}} \diamond\{i f\}\left\{\gamma^{0} q_{0}-\frac{\mathbf{q} \cdot \boldsymbol{\gamma}}{a}-\hat{M}-\Pi_{A}^{\mathrm{eq}}\right\}+i \diamond\left\{G_{R}^{\mathrm{eq}}\right\}\{i f\} \Pi_{\rho}^{\mathrm{eq}} \\
=G_{R}^{\mathrm{eq}} \widetilde{\delta \Pi_{\lessgtr}}-\widetilde{\delta G_{\lessgtr}}\left(\gamma^{0} q_{0}-\frac{\mathbf{q} \cdot \gamma}{a}-\hat{M}-\Pi_{A}^{\mathrm{eq}}\right) .
\end{gathered}
$$

By multiplying a helicity projection operator with $h= \pm 1$

$$
P_{h} \equiv \frac{1+h \mathbf{n} \cdot \boldsymbol{\sigma}}{2}, \quad \mathbf{n}=\frac{\mathbf{q}}{q}, \quad \sigma^{i}=\gamma^{0} \gamma^{i} \gamma_{5}
$$

on [(3.16)-(3.18)], and taking trace of spinors, we get

$$
\begin{aligned}
&- i \operatorname{tr}\left[P _ { h } \left(\diamond\left\{\gamma^{0} q_{0}-\frac{\mathbf{q} \cdot \gamma}{a}-\hat{M}-\Pi_{R}^{\mathrm{eq}}\right\}\{i f\} G_{\rho}^{\mathrm{eq}}-\Pi_{\rho}^{\mathrm{eq}} \diamond\{i f\}\left\{G_{A}^{\mathrm{eq}}\right\}\right.\right. \\
&\left.\left.-G_{\rho}^{\mathrm{eq}} \diamond\{i f\}\left\{\gamma^{0} q_{0}-\frac{\mathbf{q} \cdot \gamma}{a}-\hat{M}-\Pi_{A}^{\mathrm{eq}}\right\}+\diamond\left\{G_{R}^{\mathrm{eq}}\right\}\{i f\} \Pi_{\rho}^{\mathrm{eq}}\right)\right] \\
&=\operatorname{tr}\left[P_{h}\left(\left(\hat{M}+\Pi_{H}^{\mathrm{eq}}\right) \widetilde{\delta G_{\lessgtr}}-\widetilde{\delta G_{\lessgtr}}\left(\hat{M}+\Pi_{H}^{\mathrm{eq}}\right)\right)\right] \\
& \quad+\operatorname{tr}\left[P_{h}\left(\widetilde{\delta \Pi_{\lessgtr}} G_{A}^{\mathrm{eq}}+\frac{1}{2} \Pi_{\rho}^{\mathrm{eq}} \widetilde{\delta G_{\lessgtr}}-G_{R}^{\mathrm{eq}} \widetilde{\delta \Pi_{\lessgtr}}+\frac{1}{2} \widetilde{\delta G_{\lessgtr}} \Pi_{\rho}^{\mathrm{eq}}\right)\right],
\end{aligned}
$$

where $\Pi_{H}=\left(\Pi_{R}+\Pi_{A}\right) / 2$. 
We make the following quasi-particle ansatz for $\delta \widetilde{G_{\lessgtr}}$. In the present paper, we consider a situation that two RH neutrinos have almost degenerate masses. Hence their poles in the Green function can be approximated by a single pole of Breit-Wigner type:

$$
\begin{aligned}
\widetilde{\delta G_{\lessgtr}} \simeq & \sum_{h= \pm} i \delta f_{N, h}\left(q_{0}, X\right) G_{\rho}^{\mathrm{eq}} P_{h} \\
\simeq & \sum_{h= \pm}\left(-\delta f_{N, h, q}\right) \frac{\Gamma_{q}}{\left(q_{0}-\omega_{q}\right)^{2}+\Gamma_{q}^{2} / 4} \frac{q_{+}+M}{2 \omega_{q}} P_{h} \\
& +\sum_{h= \pm}\left(-\delta f_{N, h, q}^{*}\right) \frac{\Gamma_{q}}{\left(q_{0}+\omega_{q}\right)^{2}+\Gamma_{q}^{2} / 4} \frac{\not q_{-}+M}{2 \omega_{q}} P_{h},
\end{aligned}
$$

where we set the momentum at on-shell $q_{ \pm \mu}=\left( \pm \omega_{q},-\mathbf{q}\right)_{\mu}$ and

$$
G_{\rho}^{\mathrm{eq}}=\simeq \sum_{h= \pm} \frac{i 2 q_{0} \Gamma_{q}(\not d+M)}{\left(q_{0}^{2}-\omega_{q}^{2}\right)+\omega_{q}^{2} \Gamma_{q}^{2}} P_{h}
$$

is the spectral density of RH neutrino. Two mass eigenstates are summed in the distribution function $\delta f_{N}$. As explained in section 3.3, flavour off-diagonal components of the distribution function is suppressed by a cancellation of two mass eigenstates. But when the system is out-of-equilibrium, off-diagonal component of $\delta f_{N}$ becomes comparable to its diagonal one.

Also note that hermiticity of Wightman function

$$
\left[G_{<}\left(q_{0}, \mathbf{q}\right)\right]^{\dagger}=\gamma^{0} G_{<}\left(q_{0}, \mathbf{q}\right) \gamma^{0}
$$

together with spatial homogeneity and isotropy require the relation $\delta f_{N, h, q}^{\dagger}=\delta f_{N, h, q}$. Majorana condition

$$
\left[G_{<}\left(q_{0}, \mathbf{q}\right)\right]^{C}=C\left[G_{>}\left(-q_{0},-\mathbf{q}\right)\right]^{\mathrm{t}} C^{-1}=G_{<}\left(q_{0}, \mathbf{q}\right)
$$

relates the positive and negative frequency parts as in (3.21).

We then insert the ansatz of $\widetilde{\delta G_{\lessgtr}}$ of (3.21) into (3.20) and perform $q_{0}$ integration: $\int_{0}^{\infty} d q_{0} / 2 \pi$. It is dominated near the region $q_{0} \sim \omega_{\mathbf{q}}=\sqrt{M^{2}+|\mathbf{q}|^{2}}$ (see appendix A), and we get an evolution equation for the density matrix;

$$
-i d_{t} f_{N, h, q}=-\left[\omega_{q h}^{\mathrm{eff}}, \delta f_{N, h, q}\right]+\frac{\mathcal{S}}{2}
$$

The density matrix $f_{N, h, q}$ contains an equilibrium part $f_{N, h, q}^{\mathrm{eq}}=f_{N}\left(\omega_{q}\right) \mathbf{1}_{2 \times 2}$ and a deviation from it. The derivation of the l.h.s. (the kinetic term $d_{t} f_{N, h, q}$ ) is given in appendix A. The first term of the r.h.s. in (3.20) gives an effective Hamiltonian,

$$
\omega_{q h}^{\mathrm{eff}}=\operatorname{tr}\left\{\left(\hat{M}+\Pi_{H}^{\mathrm{eq}}(q)\right) \frac{\not q+M}{2 \omega_{q}} P_{h}\right\}
$$




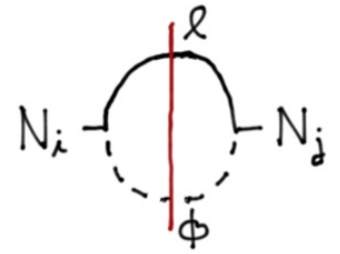

(a)

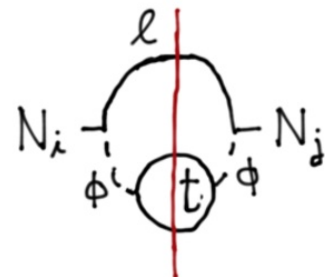

(b)

Figure 3. Two dominant contributions to the self-energy diagrams of figure 1(a). Propagators that cross with the cut-line in the middle are put on mass-shell. Internal lines are no longer full propagators. The left figure (a) gives a decay and an inverse-decay term of RH neutrinos in the $\mathrm{KB}$ equation. In the right figure (b), we consider a loop correction of the Higgs propagator by top quarks. It gives scattering terms such as $N+\bar{\ell} \leftrightarrow t+\bar{Q}$ or $N+Q \leftrightarrow \ell+t$ in the KB equation [64].

while the second term gives the collision term,

$$
\begin{aligned}
\mathcal{S}= & -\operatorname{tr}\left[P_{h}\left(\widetilde{\delta \Pi_{\lessgtr}} G_{\rho}^{\mathrm{eq}}-\Pi_{\rho}^{\mathrm{eq}} \widetilde{\delta G_{\lessgtr}}+G_{\rho}^{\mathrm{eq}} \widetilde{\delta \Pi_{\lessgtr}}-\widetilde{\delta G_{\lessgtr}} \Pi_{\rho}^{\mathrm{eq}}\right)\right] \\
= & +i \operatorname{tr}\left[P_{h}\left(\left\{\widetilde{\delta \Pi_{>}}, G_{<}^{\mathrm{eq}}\right\}+\left\{\Pi_{>}^{\mathrm{eq}}, \widetilde{\delta G_{<}}\right\}-\left\{\widetilde{\delta \Pi_{<}}, G_{>}^{\mathrm{eq}}\right\}-\left\{\Pi_{<}^{\mathrm{eq}}, \widetilde{\delta G_{>}}\right\}\right)\right] \\
= & +i\left\{\operatorname{tr}\left[P_{h} \frac{\phi+M}{2 \omega_{q}} \delta \Pi_{>}(q)\right],-f_{N, h, q}^{\mathrm{eq}}\right\}+i\left\{\operatorname{tr}\left[P_{h} \frac{\phi+M}{2 \omega_{q}} \Pi_{>}^{\mathrm{eq}}(q)\right],-\delta f_{N, h, q}\right\} \\
& -i\left\{\operatorname{tr}\left[P_{h} \frac{\not+M}{2 \omega_{q}} \delta \Pi_{<}(q)\right], 1-f_{N, h, q}^{\mathrm{eq}}\right\}-i\left\{\operatorname{tr}\left[P_{h} \frac{\not q+M}{2 \omega_{q}} \Pi_{<}^{\mathrm{eq}}(q)\right],-\delta f_{N, h, q}\right\} .
\end{aligned}
$$

Here we have used smallness of the flavour off-diagonal components $G_{i \neq j}^{\mathrm{eq}}$ in the $q_{0}$ integration (see discussion after (3.11)), and smallness of flavour dependent thermal corrections to $G_{R}$.

\section{Kinetic equation for density matrix}

In deriving kinetic equations for the density matrix, we need to make quasi-particle ansatz in (3.21). Similar ansatz must be imposed on the internal lines in the self-energy diagrams $\Pi$ because distribution functions (even when they are matrix-valued) are defined only on mass-shell. This is the most subtle point in the KB approach. In order to take various diagrams contained in each self-energy diagram in figure 1 , an often-adopted method is to expand the full propagators and cut the self-energy diagram into two. Examples are shown in figure 3. On the cut-line, on-shell propagatos are used.

\subsection{Kinetic equation for $\mathrm{RH}$ neutrinos}

The collision term (3.27) is proportional to

$$
\operatorname{tr}\left[P_{h}\left(\left\{\Pi_{>}, G_{<}\right\}-\left\{\Pi_{<}, G_{>}\right\}\right)\right] .
$$

The first term with $G_{<}$describes decay (or scattering) of RH neutrino (plus other particles) into others while the second term with $G_{>}$is an inverse-decay (or inverse scattering). By 
expanding the full propagators in the self-energy $\Pi$ and cutting the diagram into two, we have various diagrams with on-shell external lines. External lines are assigned to either incoming or outgoing particles. If a cut diagram with $G_{<}$represents a scattering process of $N+i+j \cdots \rightarrow a+b+\cdots$, it can be expressed as

$$
-\operatorname{tr}\left\{\Pi_{>}(q)(\not q+M) P_{h}\right\}=\sum_{i, \ldots, a, \ldots} \int d \Pi_{i, \ldots, a, \ldots} \gamma_{h i j \ldots}^{a b \ldots} f_{i} f_{j} \ldots\left(1-\eta_{a} f_{a}\right)\left(1-\eta_{b} f_{b}\right) \ldots
$$

$\eta_{a, i}= \pm 1$ corresponding to boson or fermion. Here the integral measure is defined as

$$
d \Pi_{i, \ldots, a, \ldots}=\prod_{i, \ldots, a, \ldots} \frac{d^{3} q_{i}}{(2 \pi)^{3} 2 \omega_{i}} \cdots \frac{d^{3} p_{a}}{(2 \pi)^{3} 2 \omega_{a}} \cdots \times(2 \pi)^{4} \delta^{(4)}\left(q+\sum_{i} q_{i}-\sum_{a} p_{a}\right)
$$

where $q$ is a momentum of incoming $\mathrm{RH}$ neutrino, $q_{i}$ and $p_{a}$ are momenta of other incoming and outgoing particles. On the other hand, if a diagram with $G_{>}$represents an inverse scattering process of $a+b+\cdots \rightarrow N+i+j+\cdots$, it can be expressed as

$$
\operatorname{tr}\left\{\Pi_{<}(q)(\not q+M) P_{h}\right\}=\sum_{i, \ldots, a, \ldots} \int d \Pi_{i, \ldots, a, \ldots} \gamma_{h i j \ldots}^{a b \ldots .}\left(1-\eta_{i} f_{i}\right)\left(1-\eta_{j} f_{j}\right) \ldots f_{a} f_{b} \ldots
$$

Combining these two contributions, the evolution equation for the density matrix $f_{N, h, q}(3.25)$ is written as

$$
\begin{aligned}
d_{t} f_{N, h, q}= & -i\left[\omega_{q s}^{\mathrm{eff}}, f_{N, h, q}\right] \\
& -\frac{1}{2} \frac{1}{2 \omega_{q}} \sum_{i, \ldots, a, \ldots} \int d \Pi_{i, \ldots, a, \ldots}\left\{\gamma_{h i j \ldots}^{a b \ldots}, f_{N, h, q}\right\} f_{i} f_{j} \ldots\left(1-\eta_{a} f_{a}\right)\left(1-\eta_{b} f_{b}\right) \ldots \\
& +\frac{1}{2} \frac{1}{2 \omega_{q}} \sum_{i, \ldots, a, \ldots} \int d \Pi_{i, \ldots, a, \ldots}\left\{\gamma_{h i j \ldots}^{a b \ldots},\left(1-f_{N, h, q}\right)\right\}\left(1-\eta_{i} f_{i}\right)\left(1-\eta_{j} f_{j}\right) \ldots f_{a} f_{b} \ldots
\end{aligned}
$$

In this expression, we combined variations as $\Pi=\Pi^{(\mathrm{eq})}+\delta \Pi$ and $f_{N}=f_{N}^{(\mathrm{eq})}+\delta f_{N}$ for notational simplicity. 0 -th order term of the variation $\delta$ automatically cancels due to the detailed balance condition in the equilibrium.

Let us now consider a specific diagram of figure 3(a). This diagram is reduced to the cut diagram of figure $4(\mathrm{a})$. Figure $4(\mathrm{~b})$ is its conjugate and $N$ decays into $\left(\bar{\ell}, \phi^{*}\right)$. Other diagrams like figure 3(b) are of higher orders in the Yukawa couplings, and we omit them in the following. From figure 3(a) and its conjugate, we have

$$
\sum_{\alpha} \int d \Pi_{p k}\left(\gamma_{h}^{\ell^{\alpha} \phi}\left(1-f_{\ell^{\alpha} p}\right)\left(1+f_{\phi k}\right)+\left(\gamma_{-h}^{\ell^{\alpha} \phi}\right)^{*}\left(1-f_{\bar{\ell}^{\alpha} p}\right)\left(1+f_{\bar{\phi} k}\right)\right)
$$

for (4.2), and

$$
\sum_{\alpha} \int d \Pi_{p k}\left(\gamma_{h}^{\ell^{\alpha} \phi} f_{\ell^{\alpha} p} f_{\phi k}+\left(\gamma_{-h}^{\ell^{\alpha} \phi}\right)^{*} f_{\bar{\ell}^{\alpha} p} f_{\bar{\phi} k}\right)
$$

for (4.4) where the following relation

$$
\gamma_{h}^{\bar{\ell}^{\alpha} \bar{\phi}}=\left(\gamma_{-h}^{\ell^{\alpha} \phi}\right)^{*}
$$




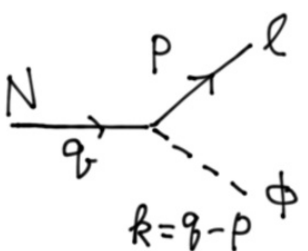

(a)

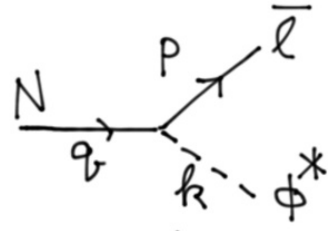

(b)

Figure 4. Decay of RH neutrino into $(\ell, \phi)$ and $\left(\bar{\ell}, \phi^{*}\right)$.

is used. The decay matrix $\gamma_{h}^{\ell^{\alpha}} \phi$ is given by

$$
\left(\gamma_{h}^{\ell^{\alpha} \phi}\right)_{i j} \equiv\left(h_{i \alpha}^{\dagger} h_{\alpha j}\right) g_{w}\left(q \cdot p-h\left(\omega_{q} \frac{\mathbf{q} \cdot \mathbf{p}}{|\mathbf{q}|}-\omega_{p}|\mathbf{q}|\right)\right)
$$

where we have used the relation

$$
\operatorname{tr}\left((\not q+M) \frac{1+h \mathbf{n} \cdot \boldsymbol{\sigma}}{2} \frac{1-\gamma^{5}}{2} \not p\right)=\left(q \cdot p-h\left(\omega_{q} \frac{\mathbf{q} \cdot \mathbf{p}}{|\mathbf{q}|}-\omega_{p}|\mathbf{q}|\right)\right) .
$$

The first term $q \cdot p$ is even under the helicity flip $h \rightarrow-h$, while the second term is odd. The integral

$$
\int \frac{d^{3} p d^{3} k}{2 \omega_{p} 2 \omega_{k}} \delta^{4}(q-p-k)\left(\omega_{q} \frac{\mathbf{q} \cdot \mathbf{p}}{|\mathbf{q}|}-\omega_{p}|\mathbf{q}|\right)
$$

vanishes when thermal effects of the SM particles, namely the thermal mass $(\sim g T)$ and the statistical factor (Pauli blocking) of leptons, are neglected.

The kinetic equaction (4.5) describes an evolution of the density matrix $f_{N}$ of the $\mathrm{RH}$ neutrinos. Since the equilibrium distribution satisfies the detailed balance condition, the r.h.s. is nonvanishing only when various quantities are out-of-equilibrium. We take a variation of (4.5) around the equilibrium. Here note that the relations $\delta f_{\ell}=-\delta f_{\bar{\ell}}$, $\delta f_{\phi}=-\delta f_{\bar{\phi}}$ hold since the SM gauge particles are in thermal equilibrium and their chemical potentials are vanishing.

In order to solve the kinetic equations, it is convenient to define helicity even and odd combinations $\delta f_{N, q}^{\text {even,odd }}$ by

$$
\delta f_{N, q}^{\text {even }} \equiv \delta f_{N,+, q}+\delta f_{N,-, q}, \quad \delta f_{N, q}^{\text {odd }} \equiv \delta f_{N,+, q}-\delta f_{N,-, q}
$$

Since helicity operator $\mathbf{n} \cdot \boldsymbol{\sigma}$ is parity-odd and RH neutrino is invariant under the charge conjugation, $\delta f_{N, q}^{\text {even,odd }}$ are CP-even and odd components respectively; in terms of these components, eq. (4.5) with the cut-diagram in figure 3(a) can be rewritten as a set of 
equations

$$
\begin{aligned}
& d_{t}\left(2 f_{N, q}^{\mathrm{eq}}+\delta f_{N, q}^{\mathrm{even}}\right) \\
& =-i\left[\frac{\omega_{q+}^{\mathrm{eff}}+\omega_{q-}^{\mathrm{eff}}}{2}, \delta f_{N, q}^{\mathrm{even}}\right]-i\left[\frac{\omega_{q+}^{\mathrm{eff}}-\omega_{q-}^{\mathrm{eff}}}{2}, \delta f_{N, q}^{\mathrm{odd}}\right] \\
& -\frac{1}{2} \frac{1}{2 \omega_{q}} \int d \Pi_{p k} \sum_{\alpha}\left\{\Re\left(\gamma_{+}^{\ell^{\alpha} \phi}+\gamma_{-}^{\ell^{\alpha} \phi}\right), \delta f_{N, q}^{\mathrm{even}}\right\}\left(1-f_{\ell^{\alpha} p}^{\mathrm{eq}}+f_{\phi k}^{\mathrm{eq}}\right) \\
& -\frac{1}{2} \frac{1}{2 \omega_{q}} \int d \Pi_{p k} \sum_{\alpha}\left\{i \Im\left(\gamma_{+}^{\ell^{\alpha} \phi}-\gamma_{-}^{\ell^{\alpha} \phi}\right), \delta f_{N q}^{\mathrm{odd}}\right\}\left(1-f_{\ell^{\alpha} p}^{\mathrm{eq}}+f_{\phi k}^{\mathrm{eq}}\right) \\
& +\frac{1}{2 \omega_{q}} \int d \Pi_{p k} \sum_{\alpha} i \Im\left(\gamma_{+}^{\ell^{\alpha} \phi}+\gamma_{-}^{\ell^{\alpha} \phi}\right)\left(\delta f_{\ell^{\alpha} p}\left(f_{\phi k}+f_{N, q}^{\mathrm{eq}}\right)+\delta f_{\phi k}\left(f_{\ell^{\alpha} p}-f_{N, q}^{\mathrm{eq}}\right)\right), \\
& d_{t}\left(\delta f_{N q}^{\text {odd }}\right) \\
& =-i\left[\frac{\omega_{q+}^{\mathrm{eff}}+\omega_{q-}^{\mathrm{eff}}}{2}, \delta f_{N q}^{\mathrm{odd}}\right]-i\left[\frac{\omega_{q+}^{\mathrm{eff}}-\omega_{q-}^{\mathrm{eff}}}{2}, \delta f_{N q}^{\mathrm{even}}\right] \\
& -\frac{1}{2} \frac{1}{2 \omega_{q}} \int d \Pi_{p k} \sum_{\alpha}\left\{\Re\left(\gamma_{+}^{\ell^{\alpha} \phi}+\gamma_{-}^{\ell^{\alpha} \phi}\right), \delta f_{N q}^{\text {odd }}\right\}\left(1-f_{\ell^{\alpha} p}^{\mathrm{eq}}+f_{\phi k}^{\mathrm{eq}}\right) \\
& -\frac{1}{2} \frac{1}{2 \omega_{q}} \int d \Pi_{p k} \sum_{\alpha}\left\{i \Im\left(\gamma_{+}^{\ell^{\alpha} \phi}-\gamma_{-}^{\ell^{\alpha} \phi}\right), \delta f_{N q}^{\mathrm{even}}\right\}\left(1-f_{\ell^{\alpha} p}^{\mathrm{eq}}+f_{\phi k}^{\mathrm{eq}}\right) \\
& +\frac{1}{2 \omega_{q}} \int d \Pi_{p k} \sum_{\alpha} \Re\left(\gamma_{+}^{\ell^{\alpha} \phi}-\gamma_{-}^{\ell^{\alpha} \phi}\right)\left(\delta f_{\ell^{\alpha} p}\left(f_{\phi k}+f_{N, q}^{\mathrm{eq}}\right)+\delta f_{\phi k}\left(f_{\ell^{\alpha} p}-f_{N, q}^{\mathrm{eq}}\right)\right) \text {. }
\end{aligned}
$$

If we can neglect the helicity odd part of the decay width $\gamma_{h}^{\ell \phi}$ as discussed in (4.11) and the backreaction from lepton asymmetry (the last terms) is dropped, these equations for $\delta f^{\text {even }}$ and $\delta f^{\text {odd }}$ are almost decoupled. Note that the helicity dependent mass term $\left(\omega_{+}-\omega_{-}\right)$is also negligible if thermal corrections are small.

The dominant source to generate deviations is the time variation of the local equilibrium distribution $d_{t} f^{\text {eq }}$, which is absent in the equation of $\delta f^{\text {odd }}$. Hence in the decoupling limit, it is sufficient to consider only the equation for for $\delta f^{\text {even }}$. In section 5.4, we obtain the $\mathrm{CP}$ violating parameter under such a condition.

\subsection{Kinetic equation for lepton number}

The evolution equation for the lepton number is similarly obtained from the KB equation. Details of the derivation is given in section 2.4 and 2.5 in [1]. $\alpha$-th flavour lepton number current is defined by

$$
\begin{aligned}
\sum_{a}\left\langle\overline{\hat{\ell}}_{a}^{\alpha}(x) \gamma^{\mu}(x) \ell_{a}^{\alpha}\right\rangle & =-\left.\sum_{a} \operatorname{tr}\left\{\gamma(x) S_{a a \lessgtr}^{\alpha \alpha}(x, y)\right\}\right|_{y=x} \\
& =-\left.g_{w} \operatorname{tr}\left\{\gamma(x) S_{\lessgtr}^{\alpha \alpha}(x, y)\right\}\right|_{y=x}
\end{aligned}
$$

where $a$ is an $\mathrm{SU}(2)$ isospin index. Around $\mathrm{TeV}$ scale, the charged Yukawa couplings distinguishing the lepton flavours are in equilibrium and the off-diagonal components of 


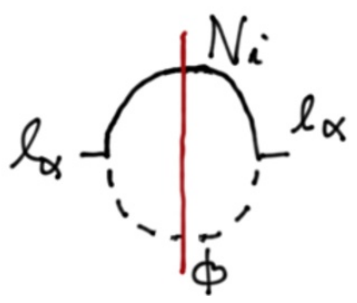

Figure 5. Cutting the self-energy diagram $\Sigma$ of leptons $\ell$. The cut diagram is the same as figure $4(\mathrm{a})$.

lepton flavour density matrix are negligible compared to diagonal ones. In the second equality, we have assumed that $\mathrm{SU}(2)$ isospin symmetry is restored.

Since the derivative expansion is an expansion of $H / \Gamma_{\ell, \phi}$, higher order terms are highly suppressed and we have

$$
\begin{aligned}
d_{t} n_{L^{\alpha}} & +3 H n_{L^{\alpha}} \\
= & g_{w} \int d \Pi_{p}\left[\operatorname{tr}\left[P_{L} \not p \Sigma_{<}^{\alpha \alpha}(p)\right]\left(1-f_{\ell^{\alpha} p}\right)+\operatorname{tr}\left[P_{L} \not p \Sigma_{>}^{\alpha \alpha}(p)\right] f_{\ell^{\alpha} p}\right. \\
& \left.-\operatorname{tr}\left[P_{L} \not \bar{\Sigma}_{<}^{\alpha \alpha}(p)\right]\left(1-f_{\bar{\ell}^{\alpha} p}\right)-\operatorname{tr}\left[P_{L} p \bar{\Sigma}_{>}^{\alpha \alpha}(p)\right] f_{\bar{\ell}^{\alpha} p}\right] .
\end{aligned}
$$

$\Sigma$ is the self-energy of the SM lepton $\ell$. If we consider, as an example, the Yukawa interaction of $(\ell, \phi, N)$, the self-energy function for leptons in figure 5 gives the same cut diagram figure 4(a). By using the same $\gamma_{h}^{\ell^{\alpha} \phi}$ in (4.9), the kinetic equation is reduced to the following Boltzmann equation;

$$
\begin{aligned}
& d_{t} n_{L^{\alpha}}+3 H n_{L^{\alpha}} \\
& =\sum_{h} \int d \Pi_{q p k}\left[\operatorname{Tr}\left[\gamma_{h}^{\ell^{\alpha} \phi}\left\{f_{N, h, q}\left(1-f_{\ell^{\alpha} p}\right)\left(1+f_{\phi k}\right)-\left(1-f_{N, h, q}\right) f_{\ell^{\alpha} p} f_{\phi k}\right\}\right]\right. \\
& \left.\quad-\operatorname{Tr}\left[\left(\gamma_{-h}^{\ell^{\alpha} \phi}\right)^{*}\left\{f_{N, h, q}\left(1-f_{\bar{\ell}^{\alpha} p}\right)\left(1+f_{\bar{\phi} k}\right)-\left(1-f_{N, h, q}\right) f_{\bar{\ell}^{\alpha} p} f_{\bar{\phi} k}\right\}\right]\right] .
\end{aligned}
$$

Here $\mathrm{Tr}$ is trace of the RH neutrino flavour.

\subsection{Kinetic equations in terms of Yield variables}

We rewrite the kinetic equations, (4.13), (4.14) and (4.17), in terms of the Yield variables $Y$ defined by

$$
Y_{N}^{\mathrm{eq}}=\frac{2}{s} \int \frac{d^{3} q}{(2 \pi)^{3}} f_{N, q}^{\mathrm{eq}}, \quad Y_{\ell^{\alpha}}^{\mathrm{eq}}=\frac{g_{w}}{s} \int \frac{d^{3} p}{(2 \pi)^{3}} f_{\ell^{\alpha} p}^{\mathrm{eq}}, \quad Y_{L^{\alpha}}=\frac{g_{w}}{s} \int \frac{d^{3} p}{(2 \pi)^{3}}\left(\delta f_{\ell^{\alpha}}-\delta f_{\bar{\ell}^{\alpha}}\right) .
$$

Here $s$ is the entropy of the universe. Note that $Y_{N}$ is a flavour matrix while $Y_{\ell}^{\alpha}$ is a c-number (or $\alpha$-th eigenvalue of a diagonal flavour matrix). In the following, we consider deviations of distribution functions of RH neutrinos $N_{i}$ and charged leptons $\ell_{\alpha}$, and other SM particles are assumed to be in the equilibrium distributions. We assume $N_{i}$ and $\ell$ are in the kinematical equilibrium. Then we can set

$$
\frac{\delta f_{N, q}^{\text {even }}}{f_{N, q}^{\mathrm{eq}}}=2 \frac{\delta Y_{N}^{\mathrm{even}}}{Y_{N}^{\mathrm{eq}}}, \quad \frac{\delta f_{N, q}^{\mathrm{odd}}}{f_{N, q}^{\mathrm{eq}}}=2 \frac{\delta Y_{N}^{\mathrm{odd}}}{Y_{N}^{\mathrm{eq}}}, \quad \frac{\delta f_{\ell^{\alpha}}}{f_{\ell^{\alpha}}^{\mathrm{eq}}}=\frac{Y_{L^{\alpha}}}{2 Y_{\ell^{\alpha}}^{\mathrm{eq}}} .
$$


Since the equations for $\delta Y$ are approximated by coupled linear differential equations, equations $(4.13),(4.14)$ can be written in a generic form with matrices $\mathrm{H}, \widetilde{\mathrm{H}}, \Gamma_{N}, \widetilde{\Gamma_{N}}, \widetilde{\Gamma_{L}}, \widetilde{\Gamma_{L}}$;

$$
\begin{aligned}
d_{t}\left(Y_{N}^{\mathrm{eq}}+\delta Y_{N}^{\mathrm{even}}\right)= & -i\left[\mathrm{H}, \delta Y_{N}^{\mathrm{even}}\right]-i\left[\widetilde{\mathrm{H}}, \delta Y_{N}^{\text {odd }}\right] \\
& -\frac{1}{2}\left\{\Gamma_{N}, \delta Y_{N}^{\text {even }}\right\}-\frac{1}{2}\left\{\widetilde{\Gamma}_{N}, \delta Y_{N}^{\text {odd }}\right\}+\sum_{\alpha} \Gamma_{L^{\alpha}} Y_{L^{\alpha}}, \\
d_{t}\left(\delta Y_{N}^{\text {odd }}\right)= & -i\left[\mathrm{H}, \delta Y_{N}^{\text {odd }}\right]-i\left[\widetilde{\mathrm{H}}, \delta Y_{N}^{\text {even }}\right] \\
& -\frac{1}{2}\left\{\Gamma_{N}, \delta Y_{N}^{\text {odd }}\right\}-\frac{1}{2}\left\{\widetilde{\Gamma}_{N}, \delta Y_{N}^{\text {even }}\right\}+\sum_{\alpha} \widetilde{\Gamma}_{L^{\alpha}} Y_{L^{\alpha}} .
\end{aligned}
$$

In the model with only Yukawa interactions, these matrices are given as follows:

$$
\begin{aligned}
\mathrm{H} & \equiv \frac{2}{s Y_{N}^{\mathrm{eq}}} \int \frac{d^{3} q}{(2 \pi)^{3}} f_{N, q}^{\mathrm{eq}} \frac{\omega_{+, q}^{\mathrm{eff}}+\omega_{-, q}^{\mathrm{eff}}}{2}, \\
\widetilde{\mathrm{H}} & \equiv \frac{2}{s Y_{N}^{\mathrm{eq}}} \int \frac{d^{3} q}{(2 \pi)^{3}} f_{N, q}^{\mathrm{eq}} \frac{\omega_{+, q}^{\mathrm{eff}}-\omega_{-, q}^{\mathrm{eff}}}{2}, \\
\Gamma_{N} & =\Re\left(\sum_{\alpha} \Gamma_{\alpha}\right), \quad \widetilde{\Gamma}_{N}=i \Im\left(\sum_{\alpha} \widetilde{\Gamma}_{\alpha}\right), \quad \Gamma_{L^{\alpha}}=i \Im\left[\Gamma_{\alpha}^{W}\right] \\
\widetilde{\Gamma}_{L^{\alpha}} & \equiv \frac{1 / s}{2 Y_{\ell^{\alpha}}^{\mathrm{eq}}} \int d \Pi_{q p k} \Re\left(\gamma_{+}^{\ell^{\alpha} \phi}-\gamma_{-}^{\ell^{\alpha} \phi}\right) f_{\ell^{\alpha} p}^{\mathrm{eq}}\left(f_{\phi k}+f_{N, q}^{\mathrm{eq}}\right),
\end{aligned}
$$

where $^{1}$

$$
\begin{aligned}
\Gamma_{\alpha} & \equiv \frac{2}{s Y_{N}^{\mathrm{eq}}} \int d \Pi_{q p k}\left(\gamma_{+}^{\ell^{\alpha} \phi}+\gamma_{-}^{\ell^{\alpha} \phi}\right) f_{N, q}^{\mathrm{eq}}\left(1-f_{\ell^{\alpha} p}^{\mathrm{eq}}+f_{\phi k}^{\mathrm{eq}}\right) \\
\widetilde{\Gamma}_{\alpha} & \equiv \frac{2}{s Y_{N}^{\mathrm{eq}}} \int d \Pi_{q p k}\left(\gamma_{+}^{\ell^{\alpha} \phi}-\gamma_{-}^{\ell^{\alpha} \phi}\right) f_{N, q}^{\mathrm{eq}}\left(1-f_{\ell^{\alpha} p}^{\mathrm{eq}}+f_{\phi k}^{\mathrm{eq}}\right) \\
\Gamma_{\alpha}^{W} & \equiv \frac{1 / s}{2 Y_{\ell^{\alpha}}^{\mathrm{eq}}} \int d \Pi_{q p k}\left(\gamma_{+}^{\ell^{\alpha} \phi}+\gamma_{-}^{\ell^{\alpha} \phi}\right) f_{\ell^{\alpha} p}^{\mathrm{eq}}\left(f_{\phi k}+f_{N q}^{\mathrm{eq}}\right) .
\end{aligned}
$$

Similarly the kinetic equation for lepton number (4.17) is also rewritten as

$$
\begin{aligned}
d_{t} Y_{L^{\alpha}}= & \operatorname{Tr}\left[2 \int d \Pi_{q p k} i \Im\left(\gamma_{+}^{\ell^{\alpha} \phi}+\gamma_{-}^{\ell^{\alpha} \phi}\right) f_{N, q}^{\mathrm{eq}}\left(1-f_{\ell^{\alpha} p}^{\mathrm{eq}}+f_{\phi k}^{\mathrm{eq}}\right) \frac{\delta Y_{N}^{\mathrm{even}}}{s Y_{N}^{\mathrm{eq}}}\right] \\
& +\operatorname{Tr}\left[2 \int d \Pi_{q p k} \Re\left(\gamma_{+}^{\ell^{\alpha} \phi}-\gamma_{-}^{\ell^{\alpha} \phi}\right) f_{N, q}^{\mathrm{eq}}\left(1-f_{\ell^{\alpha} p}^{\mathrm{eq}}+f_{\phi k}^{\mathrm{eq}}\right) \frac{\delta Y_{N}^{\mathrm{odd}}}{s Y_{N}^{\mathrm{eq}}}\right] \\
& -\left[\int d \Pi_{q p k} \operatorname{Tr}\left[\Re\left(\gamma_{+}^{\ell^{\alpha} \phi}+\gamma_{-}^{\ell^{\alpha} \phi}\right)\right] f_{N, q}^{\mathrm{eq}}\left(1+f_{\phi k}^{\mathrm{eq}}\right) \frac{Y_{L^{\alpha}}}{s 2 Y_{\ell^{\alpha}}^{\mathrm{eq}}}\right] \\
= & \operatorname{Tr}\left[i \Im\left[\Gamma_{\alpha}\right] \delta Y_{N}^{\mathrm{even}}\right]+\operatorname{Tr}\left[\Re\left[\widetilde{\Gamma}_{\alpha}\right] \delta Y_{N}^{\mathrm{odd}}\right]-\Re\left[\Gamma_{\alpha}^{W}\right] Y_{L^{\alpha}} .
\end{aligned}
$$

Hence the lepton asymmetry is generated if the r.h.s. is nonvanishing. CP violating parameter $\varepsilon$ can be read from the equation by inserting solutions of the kinetic equations for $\delta Y_{N}^{\text {even }}(4.20)$ and $\delta Y_{N}^{\text {odd }}(4.21)$.

\footnotetext{
${ }^{1}$ The real and imaginary properties of $\Gamma_{N}$ and $\widetilde{\Gamma_{N}}$ are valid when we neglect the direct $\mathrm{CP}$ violation, an interference between the tree and one-loop vertex corrections. In the resonant leptogenesis, this approximation is justified.
} 


\section{Solution of the kinetic equations}

In order to obtain the $\mathrm{CP}$ violating parameter, we solve the kinetic equations for $\delta Y_{N}$. In the derivation of the kinetic equation from the $\mathrm{KB}$ equation, we assumed that the system is not far from the local equilibrium at each time of the expanding universe. But smallness of the off-diagonal Yukawa coupling is not assumed, and the coherent flavour oscillation is fully taken into account. Since the deviation from local equilibrium is caused by the Hubble expansion, both of $\delta$ and $\partial_{t}$ are proportional to the Hubble parameter $H$. Hence we can set

$$
d_{t}\left(\delta Y_{N}^{\text {even }}\right) \simeq 0, \quad d_{t}\left(\delta Y_{N}^{\text {odd }}\right) \simeq 0
$$

in the l.h.s. of eq. (4.20), (4.21) under the condition $H \ll \Gamma_{i} \ll \Gamma_{\ell, \phi}$.

\subsection{Formal solution of $\delta Y_{N}$}

In the two-flavour case, $Y_{N}, H, \Gamma_{N}$ etc. are $2 \times 2$ matrices. We here express a $2 \times 2$ matrix $A$ as $A=\sum_{a=0}^{3}[A]^{a} \sigma^{a}$ where $\sigma^{0}=1_{2 \times 2}$ and $\sigma^{i}(i=1,2,3)$ is the Pauli matrix. Then eqs. (4.20), (4.21) are rewritten as

$$
\begin{aligned}
{\left[d_{t} Y_{N}^{\mathrm{eq}}\right]^{a} } & =C^{a b}\left[\delta Y_{N}^{\mathrm{even}}\right]^{b}+\widetilde{C}^{a b}\left[\delta Y_{N}^{\mathrm{odd}}\right]^{b}+[\mu]^{a} \\
0 & =C^{a b}\left[\delta Y_{N}^{\mathrm{odd}}\right]^{b}+\widetilde{C}^{a b}\left[\delta Y_{N}^{\mathrm{even}}\right]^{b}+[\widetilde{\mu}]^{a}
\end{aligned}
$$

where

$$
\begin{aligned}
C^{a b} & \equiv-\left(\delta^{a b}\left[\Gamma_{N}\right]^{0}+\delta_{0}^{a} \delta_{i}^{b}\left[\Gamma_{N}\right]^{i}+\delta_{i}^{a} \delta_{0}^{b}\left[\Gamma_{N}\right]^{i}+2 \delta_{i}^{a} \delta_{j}^{b} \epsilon^{i j k}[\mathrm{H}]^{k}\right), \\
\widetilde{C}^{a b} & \equiv-\left(\delta^{a b}\left[\widetilde{\Gamma}_{N}\right]^{0}+\delta_{0}^{a} \delta_{i}^{b}\left[\widetilde{\Gamma}_{N}\right]^{i}+\delta_{i}^{a} \delta_{0}^{b}\left[\widetilde{\Gamma}_{N}\right]^{i}+2 \delta_{i}^{a} \delta_{j}^{b} \epsilon^{i j k}[\widetilde{\mathrm{H}}]^{k}\right), \\
{[\mu]^{a} } & \equiv \sum_{\alpha}\left[\Gamma_{L^{\alpha}}\right]^{a} Y_{L^{\alpha}}, \quad[\widetilde{\mu}]^{a} \equiv \sum_{\alpha}\left[\widetilde{\Gamma}_{L^{\alpha}}\right]^{a} Y_{L^{\alpha}} .
\end{aligned}
$$

The Yield density matrix $Y_{N}^{(\mathrm{eq})}$ in equilibrium has an $a=0$ component only ${ }^{2}$

$$
\left[d_{t} Y_{N}^{\mathrm{eq}}\right]^{a}=\delta_{0}^{a}\left(d_{t} Y_{N}^{\mathrm{eq}}\right) .
$$

From (4.22), (4.23) and (4.24), H, $\Gamma_{N}$ and $\widetilde{\Gamma}_{L}$ (hence $\widetilde{\mu}$ ) are real matrices. Hence $\left[\Gamma_{N}\right]^{a}$, $[\mathrm{H}]^{a},[\widetilde{\mu}]^{a}$ do not have an $a=2$ component. On the other hand, $\left[\widetilde{\Gamma}_{N}\right]^{a},[\widetilde{\mathrm{H}}]^{a},[\mu]^{a}$ have only an $a=2$ component since they are imaginary matrices. ${ }^{3}$

The equations (5.2) are linear equations with respect to $\delta Y_{N}$ and can be solved in terms of the time-variation of the local equilibrium distribution $d_{t} Y_{N}^{(\mathrm{eq})}$ and the lepton asymmetry $\mu, \widetilde{\mu}$ as

$$
\left(\begin{array}{l}
{\left[\delta Y_{N}^{\text {even }}\right]} \\
{\left[\delta Y_{N}^{\text {odd }}\right]}
\end{array}\right)=\mathbf{C}^{-1}\left(\begin{array}{c}
{\left[d_{t} Y_{N}^{\mathrm{eq}}\right]-[\mu]} \\
-[\widetilde{\mu}]
\end{array}\right), \quad \mathbf{C} \equiv\left(\begin{array}{ll}
C & \widetilde{C} \\
\widetilde{C} & C
\end{array}\right) .
$$

\footnotetext{
${ }^{2}$ This statemet is correct only when we use the equilibrium distribution function for $f_{N}$. If we take higher order terms (the second term of eq. (A.5)) into account, the off-diagonal components appear and the following solutions of $\delta Y$ become more complicated.

${ }^{3}$ Flavour covariance is explicitly broken by setting the Majorana mass matrix of the $\mathrm{RH}$ neutrinos diagonal with eigenvalues $M_{1}, M_{2}$.
} 
In the expanding universe, the deviation of $\mathrm{RH}$ neutrino number densities from equilibrium $\delta Y_{N}$ is first generated and then lepton asymmetry $Y_{L}$ is generated by the flavour oscillation and decay. Here we neglect backreaction from $Y_{L}$ and evaluate the deviation of RH neutrino density directly caused by the expansion of universe. Setting $\widetilde{\mu}=0, \delta Y_{N}$ is solved as

$$
\begin{aligned}
& {\left[\delta Y_{N}^{\mathrm{even}}\right]^{a}=\left(\mathcal{C}^{-1}\right)^{a b}\left[d_{t} Y_{N}^{\mathrm{eq}}\right]^{b}=\left(\mathcal{C}^{-1}\right)^{a 0} \times d_{t} Y_{N}^{\mathrm{eq}},} \\
& {\left[\delta Y_{N}^{\mathrm{odd}}\right]^{a}=\left(\widetilde{\mathcal{C}}^{-1}\right)^{a b}\left[d_{t} Y_{N}^{\mathrm{eq}}\right]^{b}=\left(\widetilde{\mathcal{C}}^{-1}\right)^{a 0} \times d_{t} Y_{N}^{\mathrm{eq}}}
\end{aligned}
$$

where

$$
\mathbf{C}^{-1} \equiv\left(\begin{array}{ll}
\mathcal{C}^{-1} & \widetilde{\mathcal{C}}^{-1} \\
\widetilde{\mathcal{C}}^{-1} & \mathcal{C}^{-1}
\end{array}\right) .
$$

Components in the 0 -th column of $\mathbf{C}^{-1}$ are given by

$$
\begin{aligned}
\left(\mathcal{C}^{-1}\right)^{00}= & \frac{-1}{D}\left[\Gamma_{N}\right]^{0}\left\{\left(\left[\Gamma_{N}\right]^{0}\right)^{2}+4([\mathrm{H} \cdot \mathrm{H}]+[\widetilde{\mathrm{H}} \cdot \widetilde{\mathrm{H}}])\right\} \\
\left(\mathcal{C}^{-1}\right)^{i 0}= & \frac{1}{D}\left\{\left(\left[\Gamma_{N}\right]^{0}\right)^{2}\left[\Gamma_{N}\right]^{i}+4\left(\left[\Gamma_{N} \cdot \mathrm{H}\right]-\left[\widetilde{\Gamma}_{N} \cdot \widetilde{\mathrm{H}}\right]\right)[\mathrm{H}]^{i}-2\left[\Gamma_{N}\right]^{0} \epsilon^{i j k}\left[\Gamma_{N}\right]^{j}[\mathrm{H}]^{k}\right\} \\
\left(\widetilde{\mathcal{C}}^{-1}\right)^{00}= & 0 \\
\left(\widetilde{\mathcal{C}}^{-1}\right)^{i 0}= & \frac{1}{D}\left\{\left(\left[\Gamma_{N}\right]^{0}\right)^{2}\left[\widetilde{\Gamma}_{N}\right]^{i}+4\left(\left[\Gamma_{N} \cdot \mathrm{H}\right]-\left[\widetilde{\Gamma}_{N} \cdot \widetilde{\mathrm{H}}\right]\right)[\widetilde{\mathrm{H}}]^{i}\right. \\
& \left.\quad-2\left[\Gamma_{N}\right]^{0} \epsilon^{i j k}\left[\Gamma_{N}\right]^{j}[\widetilde{\mathrm{H}}]^{k}-2\left[\Gamma_{N}\right]^{0} \epsilon^{i j k}\left[\widetilde{\Gamma}_{N}\right]^{j}[\mathrm{H}]^{k}\right\}
\end{aligned}
$$

where $D$ is the determinant,

$$
\begin{aligned}
D \equiv & \left(\left[\Gamma_{N}\right]^{0}\right)^{2}\left\{\left(\left[\Gamma_{N}\right]^{0}\right)^{2}-\left[\Gamma_{N} \cdot \Gamma_{N}\right]+\left[\widetilde{\Gamma}_{N} \cdot \widetilde{\Gamma}_{N}\right]+4([\mathrm{H} \cdot \mathrm{H}]+[\widetilde{\mathrm{H}} \cdot \widetilde{\mathrm{H}}])\right\} \\
& -4\left\{\left[\Gamma_{N} \cdot \mathrm{H}\right]+\left[\widetilde{\Gamma}_{N} \cdot \widetilde{\mathrm{H}}\right]\right\}^{2} .
\end{aligned}
$$

$[\cdot]$ denotes a summation over $i=1,2,3$.

\subsection{CP-violation parameter $\varepsilon$}

In order to read the effective $C P$-violating parameter $\varepsilon$, we set $Y_{L}=0$ and insert (5.6) into the kinetic equation of the lepton numbers (4.29),

$$
\begin{aligned}
d_{t} Y_{L^{\alpha}} & =\operatorname{Tr}\left[i \Im\left(\Gamma_{\alpha}\right) \delta Y_{N}^{\text {even }}\right]+\operatorname{Tr}\left[\Re\left(\widetilde{\Gamma}_{\alpha}\right) \delta Y_{N}^{\text {odd }}\right] \\
& =2\left[\Gamma_{\alpha}\right]^{2}\left[\delta Y_{N}^{\text {even }}\right]^{2}+2 \sum_{a=0,1,3}\left[\widetilde{\Gamma}_{\alpha}\right]^{a}\left[\delta Y_{N}^{\text {odd }}\right]^{a} \\
& =2\left\{\left[\Gamma_{\alpha}\right]^{2}\left(\mathcal{C}^{-1}\right)^{20}+\left[\widetilde{\Gamma}_{\alpha}\right]^{1}\left(\widetilde{\mathcal{C}}^{-1}\right)^{10}+\left[\widetilde{\Gamma}_{\alpha}\right]^{3}\left(\widetilde{\mathcal{C}}^{-1}\right)^{30}\right\} \times d_{t} Y_{N}^{\text {eq }} \\
& =\frac{4\left[\Gamma_{N}\right]^{0}}{D} \epsilon^{i j k}\left\{\left[\Gamma_{\alpha}\right]^{i}\left[\Gamma_{N}\right]^{j}[\mathrm{H}]^{k}+\left[\widetilde{\Gamma}_{\alpha}\right]^{i}\left[\Gamma_{N}\right]^{j}[\widetilde{\mathrm{H}}]^{k}+\left[\widetilde{\Gamma}_{\alpha}\right]^{i}\left[\widetilde{\Gamma}_{N}\right]^{j}[\mathrm{H}]^{k}\right\} \times\left(-d_{t} Y_{N}^{\text {eq }}\right) .
\end{aligned}
$$


The r.h.s. can be rewritten in terms of $2\left[\delta Y_{N}\right]^{0}=\operatorname{Tr}\left(\delta Y_{N}\right)$, which is the total RH neutrino number deviated from the local equilibrium. Especially, neglecting the difference of helicity, we can write the r.h.s. of (5.10) in terms of $\left[\delta Y_{N}^{\text {even }}\right]^{0}$ in (5.6) as

$$
d_{t} Y_{L^{\alpha}}=2 \varepsilon^{\alpha}\left[\Gamma_{N}\right]^{0}\left[\delta Y_{N}^{\text {even }}\right]^{0} .
$$

Here $\left[\Gamma_{N}\right]^{0}$ is an averaged decay rate of $\mathrm{RH}$ neutrinos into charged lepton $\ell^{\alpha}$. The CPviolating parameter $\varepsilon^{\alpha}$ defined by the coefficient ${ }^{4}$ is read as

$$
\begin{aligned}
\varepsilon^{\alpha} & =\frac{2 \epsilon^{i j k}\left\{\left[\Gamma_{\alpha}\right]^{i}\left[\Gamma_{N}\right]^{j}[\mathrm{H}]^{k}+\left[\widetilde{\Gamma}_{\alpha}\right]^{i}\left[\Gamma_{N}\right]^{j}[\widetilde{\mathrm{H}}]^{k}+\left[\widetilde{\Gamma}_{\alpha}\right]^{i}\left[\widetilde{\Gamma}_{N}\right]^{j}[\mathrm{H}]^{k}\right\}}{\left(\left(\left[\Gamma_{N}\right]^{0}\right)^{2}+4([\mathrm{H} \cdot \mathrm{H}]+[\widetilde{\mathrm{H}} \cdot \widetilde{\mathrm{H}}])\right)\left[\Gamma_{N}\right]^{0}} \\
& =-i \frac{\operatorname{tr}\left(\Gamma_{\alpha} \Gamma_{N} \mathrm{H}+\widetilde{\Gamma}_{\alpha} \Gamma_{N} \widetilde{\mathrm{H}}+\widetilde{\Gamma}_{\alpha} \widetilde{\Gamma}_{N} \mathrm{H}\right)}{\left(\left(\left[\Gamma_{N}\right]^{0}\right)^{2}+4([\mathrm{H} \cdot \mathrm{H}]+[\widetilde{\mathrm{H}} \cdot \widetilde{\mathrm{H}}])\right)\left[\Gamma_{N}\right]^{0}} .
\end{aligned}
$$

The result is valid when it is justified to replace $d_{t} Y_{N}$ by its equilibrium value $d_{t} Y_{N}^{\mathrm{eq}}$. Though our calculation fixes the flavour basis in which the Majorana masses are diagonal, the final form is written in a flavour covariant way. The above definition of $\varepsilon$ is appropriate since the numerator of the ordinary definition

$$
\varepsilon \equiv \frac{\Gamma_{N \rightarrow \ell \phi}-\Gamma_{N \rightarrow \overline{\ell \phi}}}{\Gamma_{N \rightarrow \ell \phi}+\Gamma_{N \rightarrow \overline{\ell \phi}}}
$$

is replaced by $d_{t} Y_{L} / 2\left[\delta Y_{N}\right]^{0}$ while the denominator is approximated by $\Gamma_{N}$.

\subsection{Explicit forms of $\delta Y_{N}$}

In this section, we use explicit forms of various quantities to rewrite the formal expression (5.12) in a more familiar form.

$\mathrm{H}(\widetilde{\mathrm{H}})$ is the helicity even (odd) part of the mass (with thermal corrections included) and given in (4.22). $\widetilde{\mathrm{H}}$ has an $a=2$ component only. For $\mathrm{H}, a=0$ component is the total mass and decouples from the equation. $a=3$ component of $\mathrm{H}$ gives the mass difference

$$
2[\mathrm{H}]^{3}=\frac{\xi_{0}}{s Y_{N}^{\mathrm{eq}}}\left(M_{1}-M_{2}\right)+\cdots
$$

where

$$
\xi_{0} \equiv 2 M \int \frac{d q^{3}}{(2 \pi)^{3}} \frac{1}{\omega_{q}} f_{N q}^{\mathrm{eq}}
$$

\footnotetext{
${ }^{4}$ Such a definition of $\varepsilon$ was also adopted in $[65,66]$. They concluded that, since the quantity corresponding to $\left[\delta Y_{N}^{\text {even }}\right]^{2}$ oscillates with time as can be seen from eq. (4.20), the lepton asymmetry also behaves similar oscillatory behavior. Such behavior is interpreted in their analysis as an oscillating CP-violating parameter by expressing $\left[\delta Y_{N}\right]^{2}$ in terms of the non-oscillatory quantity $\left[\delta Y_{N}\right]^{0}$. In the strong washout regime, the effect of the oscillation is averaged out. The averaged CP violating parameter in the papers [65, 66] is inconsistent with ours. The discrepancy seems to be caused by neglecting one of the decay widths in their analysis, corresponding a partial truncation of the self-energy diagrams.
} 
The $\cdots$ in $[\mathrm{H}]^{3}$ represents finite temperature (and density) corrections to the RH neutrino potential. Off-diagonal components $[\mathrm{H}]^{1}$ and $[\widetilde{\mathrm{H}}]^{2}$ represent kinetic mixing induced by the thermal effects, and can be removed by flavour rotation at each time. Unitary matrix diagonalizing the mass matrix is time dependent, but in the following analysis, we neglect time-dependence of the thermal mass and mixing. If we neglect the statistical effects, the coefficient in $[H]^{3}$ is given by $\left(\xi_{0} / s Y_{N}^{\mathrm{eq}}\right)=K_{1}(M / T) / K_{2}(M / T)$. At low temperature $T \ll M$ it approaches $\left(\xi_{0} / s Y_{N}^{\mathrm{eq}}\right) \rightarrow 1$ while at high temperature $T \gg M$, it behaves as $\left(\xi_{0} / s Y_{N}^{\mathrm{eq}}\right) \sim M /(2 T)$

$\Gamma_{N}$ comes from the self-energy diagrams of $\mathrm{RH}$ neutrinos, and contains information of (inverse) decay or scattering of RH neutrinos. We decompose $\Gamma_{N}$ into $\Gamma_{\alpha}$ by fixing the flavour $\alpha$ of lepton $\ell^{\alpha}$ in the final state. Only the real part appears in the KB equation. From (4.23), we can decompose $\Gamma_{N}$ in the model (2.2) as

$$
\Gamma_{N}=\frac{\xi}{s Y_{N}^{\mathrm{eq}}} \frac{\Re\left(h^{\dagger} h\right) M}{8 \pi}+\Gamma_{N}^{\mathrm{scatt}}+\Gamma_{N}^{\mathrm{vertex}}
$$

where

$$
\xi \equiv 32 \pi\left(M-\frac{m_{\phi}^{2}-m_{\ell}^{2}}{M}\right) \int d \Pi_{N \ell^{\alpha} \phi} f_{N q}^{\mathrm{eq}}\left(1-f_{\ell p}^{\mathrm{eq}}+f_{\phi k}^{\mathrm{eq}}\right) .
$$

$\Gamma_{\alpha}$ is a partial decay width that $\mathrm{RH}$ neutrino decays into $\ell^{\alpha}$. At the leading order, it is given by replacing $\left(h^{\dagger} h\right)_{i j}$ in $(5.16)$ by $\left(h_{i \alpha}^{\dagger} h_{\alpha j}\right)$ (no summation over $\alpha$ ).

The first term of $\Gamma_{N}$ is the decay amplitude at the tree level and if we neglect the statistical effects and the thermal mass of the Higgs and lepton, $\xi$ coincides with $\xi_{0}$, and approaches

$$
\left(\xi / s Y_{N}^{\mathrm{eq}}\right)=\left(\xi_{0} / s Y_{N}^{\mathrm{eq}}\right) \rightarrow M /(2 T)
$$

at high temperature. $\Gamma_{N}^{\text {scatt }}$ are corrections to the decay rate from scattering with the top quarks or gauge particles in the thermal media. $\Gamma_{N}^{\mathrm{vertex}}$ are corrections to the vertex diagram. It is negligible compared to the first term. In the resonant leptogenesis, the direct $\mathrm{CP}$ violating parameter associated with an interference between the tree and the vertex correction can be neglected compared to the indirect $\mathrm{CP}$ violation through the flavour oscillation. Then the relations $\left[\Gamma_{N}\right]^{2}=\left[\widetilde{\Gamma}_{N}\right]^{0,1,3}=0$ hold. (See footnote 1.)

In order to simplify the notation, we write

$$
\left(\Gamma_{N}\right)_{i j}=\frac{\xi_{0}}{s Y_{N}^{\mathrm{eq}}} \Gamma_{i j}^{\mathrm{eff}}, \quad\left(\widetilde{\Gamma}_{N}\right)_{i j}=\frac{\xi_{0}}{s Y_{N}^{\mathrm{eq}}} \widetilde{\Gamma}_{i j}^{\mathrm{eff}}
$$

where $\Gamma_{i j}^{\mathrm{eff}}$ and $\widetilde{\Gamma}_{i j}^{\mathrm{eff}}$ are effective decay rates including not only thermal effects but also scattering contributions. If interactions do not change the flavour structure, the effective decay matrix is written as

$$
\Gamma_{i j}^{\mathrm{eff}}=(1+\alpha) M \frac{\Re\left(h^{\dagger} h\right)_{i j}}{8 \pi}, \quad \widetilde{\Gamma}_{i j}^{\mathrm{eff}}=\tilde{\alpha} M \frac{i \Im\left(h^{\dagger} h\right)_{i j}}{8 \pi},
$$

for $a=1,2,3$ component. Furthermore, if we consider flavour independent interactions such as $B-L$ gauge interaction of $\mathrm{RH}$ neutrinos, an additional contribution is added 
to $a=0$ component $\left[\Gamma_{N}\right]^{0}$. In the following, we neglect this contribution for simplicity. When we neglect thermal effects and scattering contributions, $\alpha$ and $\widetilde{\alpha}$ vanish and diagonal components of $\Gamma_{i i}^{\mathrm{eff}}$ are reduced to the tree-level vacuum decay rate $\Gamma_{i}^{\mathrm{vac}} \equiv\left(h^{\dagger} h\right)_{i i} M /(8 \pi)$. In the following we write $\Gamma_{i}=\Gamma_{i i}^{\text {eff }}$ as a decay rate including the above corrections.

Using these quantities of $\mathrm{H}$ and $\Gamma_{N}$, we can express each component of the inverse matrix $\mathcal{C}^{-1}$ in terms of masses $M_{i}$ and decay rates $\Gamma_{i}$. The explicit forms are written in appendix B.

By using the explicit forms of $\mathcal{C}^{-1}$ in appendix B, we can write down each component of $\delta Y$ as follows. First, the diagonal components of $\delta Y_{N}^{\text {even }}(a=0,3)$ are given by

$$
\begin{aligned}
{\left[\delta Y_{N}^{\mathrm{even}}\right]^{0} } & =-\frac{d_{t} Y_{N}^{\mathrm{eq}}}{\xi_{0} /\left(s Y_{N}^{\mathrm{eq}}\right)} \frac{\Gamma_{1}+\Gamma_{2}}{2 \Gamma_{1} \Gamma_{2}} U, \\
{\left[\delta Y_{N}^{\mathrm{even}}\right]^{3} } & =-\frac{d_{t} Y_{N}^{\mathrm{eq}}}{\xi_{0} /\left(s Y_{N}^{\mathrm{eq}}\right)} \frac{-\Gamma_{1}+\Gamma_{2}}{2 \Gamma_{1} \Gamma_{2}} U
\end{aligned}
$$

where

$$
U \equiv \frac{\left(M_{1}^{2}-M_{2}^{2}\right)^{2}+M^{2}\left(\Gamma_{1}+\Gamma_{2}\right)^{2}}{\left(M_{1}^{2}-M_{2}^{2}\right)^{2}+M^{2}\left(\Gamma_{1}+\Gamma_{2}\right)^{2} X},
$$

and

$$
X=\frac{\operatorname{det}\left[\Re\left(h^{\dagger} h\right)\right](1+\alpha)^{2}-\left(\widetilde{\alpha} \Im\left(h^{\dagger} h\right)\right)^{2}}{\left(h^{\dagger} h\right)_{11}\left(h^{\dagger} h\right)_{22}(1+\alpha)^{2}} .
$$

$\left[\delta Y_{N}^{\text {even }}\right]^{0}$ gives an averaged number of the $\mathrm{RH}$ neutrinos deviated from the local equilibrium. Equivalently, $i i$-component of the matrix $\delta Y_{N}^{\text {even }}$ is given by

$$
\left(\delta Y_{N}^{\mathrm{even}}\right)_{i i}=\left[\delta Y_{N}^{\mathrm{even}}\right]^{0} \pm\left[\delta Y_{N}^{\mathrm{even}}\right]^{3}=-\frac{d_{t} Y_{N}^{\mathrm{eq}}}{\xi_{0} /\left(s Y_{N}^{\mathrm{eq}}\right)} \frac{U}{\Gamma_{i}}
$$

where \pm represents $i=1,2$ respectively.

Off-diagonal components can be similarly obtained. The real part $a=1$ and the imaginary part $a=2$ of $\delta Y_{N}^{\text {even }}$ are given by

$$
\begin{aligned}
& {\left[\delta Y_{N}^{\text {even }}\right]^{1}=\Re \delta Y_{N 12}^{\text {even }}=-2(1+\alpha) \Re\left[h^{\dagger} h\right]_{12}\left(\Gamma_{1}+\Gamma_{2}\right) M V\left[\delta Y_{N}^{\text {even }}\right]^{0},} \\
& {\left[\delta Y_{N}^{\text {even }}\right]^{2}=-\Im \delta Y_{N 12}^{\text {even }}=-2(1+\alpha) \Re\left[h^{\dagger} h\right]_{12}\left(M_{1}^{2}-M_{2}^{2}\right) V\left[\delta Y_{N}^{\text {even }}\right]^{0} .}
\end{aligned}
$$

For $\delta Y_{N}^{\text {odd }}$, we have

$$
\begin{aligned}
& {\left[\delta Y_{N}^{\text {odd }}\right]^{1}=\Re \delta Y_{N 12}^{\text {even }}=2 \widetilde{\alpha} \Im\left[h^{\dagger} h\right]_{12}\left(\Gamma_{1}+\Gamma_{2}\right) M V\left[\delta Y_{N}^{\text {even }}\right]^{0},} \\
& {\left[\delta Y_{N}^{\text {oddd }}\right]^{2}=-\Im \delta Y_{N 12}^{\text {even }}=-2 \widetilde{\alpha} \Im\left[h^{\dagger} h\right]_{12}\left(M_{1}^{2}-M_{2}^{2}\right) V\left[\delta Y_{N}^{\text {even }}\right]^{0} .}
\end{aligned}
$$

Here we defined

$$
V \equiv \frac{M^{2} /(8 \pi)}{\left(M_{1}^{2}-M_{2}^{2}\right)^{2}+M^{2}\left(\Gamma_{1}+\Gamma_{2}\right)^{2}} .
$$


$\left[\delta Y_{N}^{\text {even }}\right]^{2}$ and $\left[\delta Y_{N}^{\text {odd }}\right]^{1}$ give the $\mathrm{CP}$ violating parameter $\varepsilon$. It is given in a simplified case in the next section.

We comment on a situation when $\operatorname{det}\left[\Re\left(h^{\dagger} h\right)\right]$ becomes small. (For simplicity we set $\tilde{\alpha}=0$.) Then $X$ and accordingly $\left[\delta Y_{N}^{\text {even }}\right]^{0}$ is largely enhanced. The situation corresponds to a case that an effective decay rate (cf. (4.20)) is small. Especially when the mass difference vanishes $M_{1}=M_{2}$, it diverges at $\operatorname{det}\left[\Re\left(h^{\dagger} h\right)\right]=0$, namely when $\operatorname{det} C=0$. In such a situation, the deviation of RH neutrino number density becomes large and the assumption of our investigation, smallness of the deviation from local equilibrium, becomes invalid.

\subsection{CP violating parameter $\varepsilon$ when $\widetilde{\mathcal{C}}=\mathbf{0}$}

Finally we write the formal expression of (5.12) in a more familiar form by introducing further simplifications. We neglect the thermal mass of leptons and drop the Pauli blocking terms. Then the helicity odd part of $\gamma_{h}^{\ell \phi}$ disappears as explained in (4.11) and the offdiagonal components $\widetilde{\mathcal{C}}$ connecting the $\mathrm{CP}$ even and odd parts in $\delta Y$ vanish. Furthermore we use the vacuum value of $\Gamma_{N}(\alpha=\widetilde{\alpha}=0)$. Then, by using explicit forms of $\mathrm{H}$ in (5.14) and $\Gamma_{N}$ in (5.19) with $\Gamma_{i j}^{\mathrm{eff}}=\Gamma_{i j}^{\mathrm{vac}}$, the $\mathrm{CP}$-violating parameter $\varepsilon^{\alpha}$ is given by

$$
\begin{aligned}
\varepsilon^{\alpha} & =\frac{2 \epsilon^{i j k}\left[\Gamma_{\alpha}\right]^{i}\left[\Gamma_{N}\right]^{j}[\mathrm{H}]^{k}}{\left(\left[\Gamma_{N}\right]^{0}\right)^{2}+4[\mathrm{H} \cdot \mathrm{H}]} \\
& =\frac{2 \Re\left(h^{\dagger} h\right)_{12} \Im\left(h_{1 \alpha}^{\dagger} h_{\alpha 2}\right)}{\left(\left(h^{\dagger} h\right)_{11}+\left(h^{\dagger} h\right)_{22}\right)^{2} / 4} \frac{\left(M_{1}^{2}-M_{2}^{2}\right) M\left(\Gamma_{1}+\Gamma_{2}\right) / 2}{\left(M_{1}^{2}-M_{2}^{2}\right)^{2}+M^{2}\left(\Gamma_{1}+\Gamma_{2}\right)^{2}} .
\end{aligned}
$$

This CP violating parameter has the regulator $M^{2}\left(\Gamma_{1}+\Gamma_{2}\right)^{2}$ which is consistent with our previous result [1]. In the previous analysis we obtained the same result under an assumption that the off-diagonal Yukawa couplings are smaller than the diagonal ones. In the present analysis, we do not use such a condition, and take effects of coherent flavour oscillation fully into account. The decay widths $\Gamma_{i}^{\text {eff }}$ are determined by the effective decay width (5.19), which are obtained from the 1PI self-energy diagrams $\Pi$ by cutting the diagrams and putting external lines on mass-shell.

Finally we note that we can decompose the r.h.s. of (5.11) into $N_{i}(i=1,2)$ as

$$
d_{t} Y_{L^{\alpha}}=\sum_{i=1,2} \varepsilon_{i}^{\alpha}\left(\Gamma_{N}\right)_{i i}\left(\delta Y_{N}^{\mathrm{even}}\right)_{i i}
$$

where we define the CP violating parameter of each $N_{i}$ as

$$
\varepsilon_{i}^{\alpha}=\frac{2 \Re\left(h^{\dagger} h\right)_{12} \Im\left(h_{1 \alpha}^{\dagger} h_{\alpha 2}\right)}{\left(h^{\dagger} h\right)_{11}\left(h^{\dagger} h\right)_{22}} \frac{\left(M_{1}^{2}-M_{2}^{2}\right) M \Gamma_{j(\neq i)}}{\left(M_{1}^{2}-M_{2}^{2}\right)^{2}+M^{2}\left(\Gamma_{1}+\Gamma_{2}\right)^{2}} .
$$

When $i=1, j$ takes 2 , and vice-versa. Such a separation into a different flavour of RH neutrinos is, of course, valid only when the off-diagonal component $\left(h^{\dagger} h\right)$ is smaller than the diagonal one. The numerator of the first factor can be rewritten as

$$
2 \Re\left(h^{\dagger} h\right)_{12} \Im\left(h_{1 \alpha}^{\dagger} h_{\alpha 2}\right)=\Im\left[\left(h^{\dagger} h\right)_{12}\left(h_{1 \alpha}^{\dagger} h_{\alpha 2}\right)\right]+\Im\left[\left(h^{\dagger} h\right)_{21}\left(h_{1 \alpha}^{\dagger} h_{\alpha 2}\right)\right]
$$

which gives a consistent result with [5]. 


\section{Summary}

In the paper, we solved the KB equation without assuming that the off-diagonal component of the Yukawa couplings are small compared to the diagonal ones. In order to solve it, we first derive the kinetic equation for the density matrix. The differential equation can be reduced to a linear equation if the background is slowly changing and the deviation of the distribution function from local equilibrium is small. Then the density matrix of $\mathrm{RH}$ neutrino can be solved in terms of the time variation of the equilibrium distribution function and the generated lepton asymmetry. Its off-diagonal component determines the CP violating parameter $\varepsilon$. It is resonantly enhanced due to the almost degenerate Majorana masses and the regulator of $\varepsilon$ is given by $R_{i j}=M_{i} \Gamma_{i}+M_{j} \Gamma_{j}$. In the 2PI formalism, the decay width $\Gamma_{i}$ is given by the imaginary part of the self-energy function of the $\mathrm{RH}$ neutrinos. In addition to the loop corrections of the vertex functions, scattering effects with particles in medium are contained. The effect of coherent oscillation is fully taken into account by considering the density matrix formalism.

The derivation of the kinetic equation of the density matrix from the KB equation is based on an assumption that the distribution function is not far from the local equilibrium. It will be interesting to obtain the kinetic equation when the system is far from equilibrium. We want to come back to this problem in near future.

Note added. During the final stage of writing the manuscript, an interesting paper [80] appeared. In the paper, the authors derived the kinetic equation of density matrix based on the Hamiltonian approach, and solve the equation to obtain $\delta Y_{N}^{\text {even }}$ in the flavour covariant way. The result is consistent with ours but the interpretation of the $\mathrm{CP}$ violating parameter seems to be different. Also, in [80], the one-loop resummed effective Yukawa coupling is used to define decay and inverse-decay amplitudes ( $\Gamma_{N}$ in our notation), in which the effect of coherent oscillation is included in their analysis. In our approach based on the 2PI formalism, $\Gamma_{N}$ comes from 1PI self-energies and the effect of coherent oscillation is not contained. The indirect $\mathrm{CP}$ violating parameter $\varepsilon$ generated by resummation of $\mathrm{RH}$ neutrino propagators is taken into account by considering the multi-flavour formulation of density matrix.

\section{Acknowledgments}

We would like to thank Masato Yamanaka for discussions. This work is supported in part by Grant-in-Aid for Scientific Research (No. 23244057, 23540329) from the Japan Society for the Promotion of Science, and "The Center for the Promotion of Integrated Sciences (CPIS)" of Sokendai.

\section{A Derivation of the kinetic term $d_{t} f_{N}$}

In this appendix, we show how the the kinetic term in $(3.25)-i d_{t} f_{N, h, q}$ is derived from the 1.h.s. in (3.20):

$$
\begin{aligned}
&-i \operatorname{tr} {\left[P _ { h } \left(\diamond\left\{\gamma^{0} q_{0}-\frac{\mathbf{q} \cdot \boldsymbol{\gamma}}{a}-\hat{M}-\Pi_{R}^{\mathrm{eq}}\right\}\{i f\} G_{\rho}^{\mathrm{eq}}-\Pi_{\rho}^{\mathrm{eq}} \diamond\{i f\}\left\{G_{A}^{\mathrm{eq}}\right\}\right.\right.} \\
&\left.\left.-G_{\rho}^{\mathrm{eq}} \diamond\{i f\}\left\{\gamma^{0} q_{0}-\frac{\mathbf{q} \cdot \gamma}{a}-\hat{M}-\Pi_{A}^{\mathrm{eq}}\right\}+\diamond\left\{G_{R}^{\mathrm{eq}}\right\}\{i f\} \Pi_{\rho}^{\mathrm{eq}}\right)\right] .
\end{aligned}
$$


First we look at the leading term. For simplicity, we drop the self-energy correction $\Pi_{R}^{\mathrm{eq}}$. Then we have

$$
\begin{aligned}
i \operatorname{tr} & {\left[P_{h} \sum_{h^{\prime}} \diamond\left\{\gamma^{0} q_{0}-\frac{\mathbf{q} \cdot \gamma}{a}-\hat{M}\right\}\left\{i f_{h^{\prime}}^{\mathrm{eq}}\right\}(\not q+M) P_{h^{\prime}}\right] \frac{\Gamma_{a}}{\left(\left(q_{0}-\omega_{q}\right)^{2}+\Gamma_{q}^{2} / 4\right)} } \\
& =q_{0}\left(\partial_{X} f_{h}^{\mathrm{eq}}\left(q_{0}, X\right)-\frac{H|\mathbf{q}|^{2}}{q_{0} a^{2}} \partial_{q_{0}} f_{h}^{\mathrm{eq}}\left(q_{0}, X\right)\right) \frac{\Gamma_{a}}{\left(\left(q_{0}-\omega_{q}\right)^{2}+\Gamma_{q}^{2} / 4\right)} .
\end{aligned}
$$

If we set $q_{0}=\omega_{q}$, two terms in the bracket give a total derivative

$$
d_{t}=\left(\partial_{t} T\right) \partial_{T}+\left(\partial_{t} \omega_{q}\right) \partial_{\omega_{q}}
$$

of the on-shell Fermi distribution function $f_{h q}^{\text {eq }} \equiv f^{\text {eq }}\left(t, \omega_{q}(t)\right)$ in equilibrium. But the propagator has a Lorentz type structure and $q_{0}$ is extended around the position of the pole $q_{0}=\omega_{q}$.

We then take an effect of the remaining terms in (A.1). These terms can be rewritten as

$$
\begin{aligned}
& i \operatorname{tr}\left\{P_{h} \Pi_{\rho} \diamond\left\{i f^{\text {eq }}\right\}\left\{G_{A}\right\}-P_{h} \diamond\left\{G_{R}\right\}\left\{i f^{\text {eq }}\right\} \Pi_{\rho}\right\} \\
& \quad=i \operatorname{tr}\left\{P_{h} \Pi_{\rho} G_{A} \diamond\left\{i f^{\text {eq }}\right\}\left\{G_{A}^{-1}\right\} G_{A}-P_{\mathrm{h}} G_{R} \diamond\left\{G_{R}^{-1}\right\}\left\{i f^{\mathrm{eq}}\right\} G_{R} \Pi_{\rho}\right\} \\
& \quad \simeq-\operatorname{tr}\left\{P_{h} \diamond\left\{\gamma^{0} q_{0}-\frac{\mathbf{q} \cdot \gamma}{a}-\hat{M}\right\}\left\{f^{\mathrm{eq}}\right\}\left(G_{R} \Pi_{\rho} G_{R}+G_{A} \Pi_{\rho} G_{A}\right)\right\} .
\end{aligned}
$$

In the first equality, we have used the relation $\diamond\{f\}\{A\}=-\diamond\{A\}\{f\}$ and $\diamond\{f\}\{A\}=$ $A \diamond\{f\}\left\{A^{-1}\right\} A$ for a given matrix $A$. In the second line, we have used $G_{R / A}^{-1}=-(\not q-\hat{M}-$ $\left.\Pi_{R / A}\right)$ and droped next-to-leading order contributions $\Pi_{R, A}$.

Using (A.4), four terms in (A.1) are combined to become

$$
\begin{aligned}
2 \operatorname{tr} & \left\{P_{h} \diamond\left\{\gamma^{0} q_{0}-\frac{\mathbf{q} \cdot \boldsymbol{\gamma}}{a}-\hat{M}\right\}\{f\} G_{\rho}\right\} \\
-\operatorname{tr} & \left\{P_{h} \diamond\left\{\gamma^{0} q_{0}-\frac{\mathbf{q} \cdot \gamma}{a}-\hat{M}\right\}\left\{f^{\mathrm{eq}}\right\}\left(G_{R} \Pi_{\rho} G_{R}+G_{A} \Pi_{\rho} G_{A}\right)\right\} \\
\simeq & \left(\partial_{X} f_{h}\left(q_{0}, X\right)-\frac{H|\mathbf{q}|^{2}}{q_{0} a^{2}} \partial_{q_{0}} f_{h}\left(q_{0}, X\right)\right) \times(-i) \\
& \times\left(\frac{\Gamma_{q}}{\left(q_{0}-\omega_{q}\right)^{2}+\Gamma_{q}^{2} / 4}-\frac{\Gamma_{q}\left(q_{0}-\omega_{q}-i \Gamma_{q} / 2\right)^{2}}{2\left(\left(q_{0}-\omega_{q}\right)^{2}+\Gamma_{q}^{2} / 4\right)^{2}}-\frac{\Gamma_{q}\left(q_{0}-\omega_{q}+i \Gamma_{q} / 2\right)^{2}}{2\left(\left(q_{0}-\omega_{q}\right)^{2}+\Gamma_{q}^{2} / 4\right)^{2}}\right) \\
= & -i\left(\partial_{X} f_{h}\left(q_{0}, X\right)-\frac{H|\mathbf{q}|^{2}}{q_{0} a^{2}} \partial_{q_{0}} f_{h}\left(q_{0}, X\right)\right) \times \frac{\Gamma_{q}^{3} / 2}{\left(\left(q_{0}-\omega_{q}\right)^{2}+\Gamma_{q}^{2} / 4\right)^{2}}
\end{aligned}
$$

around the position of the pole $q_{0}=\omega_{q}$. Here, we used the approximate form $\Pi_{\rho} \sim$ $\not q \times\left(-i \omega_{q_{0}} \Gamma_{q} / M^{2}\right)$ and dropped higher order terms with respect to $\left(q_{0}-\omega_{q}\right)$. Hence, the original Lorentz type distribution becomes to have a sharper spectrum after adding the higher order terms in the KB equation. Namely, the term $\Gamma_{q}^{3} / 2 /\left(\left(q_{0}-\omega_{q}\right)^{2}+\Gamma_{q}^{2} / 4\right)^{2}$ approaches Dirac delta function $2 \pi \delta\left(q_{0}-\omega_{q}\right)$ much faster than the usual Lorentz type form $\Gamma_{a} /\left(\left(q_{0}-\omega_{q}\right)^{2}+\Gamma_{q}^{2} / 4\right)$ in the limit $\Gamma_{q} \rightarrow 0$ [81].

In this appendix, we considered a single flavour case in order to see that the distribution function is sharpened as above. The effect of flavour mixing due to the second term in (A.5) may change the flavour structure in the 1.h.s. of the kinetic equation. We want to come back to this interesting issue in future. 


\section{B Explicit forms of $\mathcal{C}^{-1}$ and $\widetilde{\mathcal{C}}^{-1}$}

In this appendix, we show explicit forms of $\mathcal{C}^{-1}$ and $\widetilde{\mathcal{C}}^{-1}$ used in the section 5.3. For brevity, we write each coefficient of the $2 \times 2$ matrices $\Gamma^{\mathrm{eff}}$ and $\widetilde{\Gamma}^{\mathrm{eff}}$ expanded in terms of $\left(1_{2 \times 2}, \sigma^{a}\right)$ as $[\Gamma]^{a}$ and $[\widetilde{\Gamma}]^{a}$ without the supersctipt "eff".

$$
\begin{gathered}
\left(\mathcal{C}^{-1}\right)^{a 0}=\frac{-1}{D}\left(\begin{array}{c}
{\left[\Gamma_{N}\right]^{0}\left\{\left(\left[\Gamma_{N}\right]^{0}\right)^{2}+\left(2[\mathrm{H}]^{3}\right)^{2}\right\}} \\
-\left(\left[\Gamma_{N}\right]^{0}\right)^{2}\left[\Gamma_{N}\right]^{1} \\
-2[\mathrm{H}]^{3}\left[\Gamma_{N}\right]^{0}\left[\Gamma_{N}\right]^{1} \\
-\left[\Gamma_{N}\right]^{3}\left\{\left(\left[\Gamma_{N}\right]^{0}\right)^{2}+\left(2[\mathrm{H}]^{3}\right)^{2}\right\}
\end{array}\right){ }^{a} \\
=\frac{-\xi_{0}^{3}}{D\left(s Y_{N}^{\mathrm{eq}}\right)^{3}}\left(\begin{array}{c}
{[\Gamma]^{0}\left\{\left([\Gamma]^{0}\right)^{2}+\left(M_{1}-M_{2}\right)^{2}\right\}} \\
-\left([\Gamma]^{0}\right)^{2}[\Gamma]^{1} \\
-\left(M_{1}-M_{2}\right)[\Gamma]^{0}[\Gamma]^{1} \\
-[\Gamma]^{3}\left\{\left([\Gamma]^{0}\right)^{2}+\left(M_{1}-M_{2}\right)^{2}\right\}
\end{array}\right), \\
\left(\widetilde{\mathcal{C}}^{-1}\right)^{a 0}=\frac{-1}{D}\left(\begin{array}{c}
+2[\mathrm{H}]^{3}\left[\Gamma_{N}\right]^{0}\left[\widetilde{\Gamma}_{N}\right]^{2} \\
-\left(\left[\Gamma_{N}\right]^{0}\right)^{2}\left[\widetilde{\Gamma}_{N}\right]^{2} \\
0
\end{array}\right)^{a}=\frac{-\xi_{0}^{3}}{D\left(s Y_{N}^{\mathrm{eq}}\right)^{3}}\left(\begin{array}{c}
+\left(M_{1}-M_{2}\right)[\Gamma]^{0}[\widetilde{\Gamma}]^{2} \\
-\left([\Gamma]^{0}\right)^{2}[\widetilde{\Gamma}]^{2} \\
0
\end{array}\right)^{a}
\end{gathered}
$$

where determinant $D$ is given by

$$
\begin{aligned}
D & =\left\{\left(\left[\Gamma_{N}\right]^{0}\right)^{2}-\left(\left[\Gamma_{N}\right]^{3}\right)^{2}\right\}\left[\left(2[\mathrm{H}]^{3}\right)^{2}+\left(\left[\Gamma_{N}\right]^{0}\right)^{2} \frac{\left(\left[\Gamma_{N}\right]^{0}\right)^{2}-\left[\Gamma_{N} \cdot \Gamma_{N}\right]-\left[\widetilde{\Gamma}_{N} \cdot \widetilde{\Gamma}_{N}\right]}{\left(\left[\Gamma_{N}\right]^{0}\right)^{2}-\left(\left[\Gamma_{N}\right]^{3}\right)^{2}}\right] \\
& =\frac{\xi_{0}^{4}}{\left(s Y_{N}^{\text {eq }}\right)^{4}} \Gamma_{1} \Gamma_{2}\left[\left(M_{1}-M_{2}\right)^{2}+\left([\Gamma]^{0}\right)^{2} \frac{\operatorname{det}\{\Gamma\}-\left([\widetilde{\Gamma}]^{2}\right)^{2}}{\Gamma_{1} \Gamma_{2}}\right]
\end{aligned}
$$

Open Access. This article is distributed under the terms of the Creative Commons Attribution License (CC-BY 4.0), which permits any use, distribution and reproduction in any medium, provided the original author(s) and source are credited.

\section{References}

[1] S. Iso, K. Shimada and M. Yamanaka, Kadanoff-Baym approach to the thermal resonant leptogenesis, JHEP 04 (2014) 062 [arXiv: 1312.7680] [INSPIRE].

[2] M. Fukugita and T. Yanagida, Baryogenesis Without Grand Unification, Phys. Lett. B 174 (1986) 45 [INSPIRE].

[3] C.S. Fong, E. Nardi and A. Riotto, Leptogenesis in the Universe, Adv. High Energy Phys. 2012 (2012) 158303 [arXiv:1301.3062] [INSPIRE].

[4] S. Davidson and A. Ibarra, A lower bound on the right-handed neutrino mass from leptogenesis, Phys. Lett. B 535 (2002) 25 [hep-ph/0202239] [INSPIRE].

[5] L. Covi, E. Roulet and F. Vissani, CP violating decays in leptogenesis scenarios, Phys. Lett. B 384 (1996) 169 [hep-ph/9605319] [INSPIRE].

[6] L. Covi and E. Roulet, Baryogenesis from mixed particle decays, Phys. Lett. B 399 (1997) 113 [hep-ph/9611425] [INSPIRE].

[7] A. Pilaftsis, CP violation and baryogenesis due to heavy Majorana neutrinos, Phys. Rev. D 56 (1997) 5431 [hep-ph/9707235] [INSPIRE]. 
[8] A. Pilaftsis and T.E.J. Underwood, Resonant leptogenesis, Nucl. Phys. B 692 (2004) 303 [hep-ph/0309342] [INSPIRE].

[9] A. Pilaftsis and T.E.J. Underwood, Electroweak-scale resonant leptogenesis, Phys. Rev. D 72 (2005) 113001 [hep-ph/0506107] [INSPIRE].

[10] F.F. Deppisch and A. Pilaftsis, Lepton Flavour Violation and $\theta_{13}$ in Minimal Resonant Leptogenesis, Phys. Rev. D 83 (2011) 076007 [arXiv:1012.1834] [INSPIRE].

[11] T. Hambye, Leptogenesis at the TeV scale, Nucl. Phys. B 633 (2002) 171 [hep-ph/0111089] [INSPIRE].

[12] S. Dar, S.J. Huber, V.N. Senoguz and Q. Shafi, TeV scale leptogenesis with heavy neutrinos, Phys. Rev. D 69 (2004) 077701 [hep-ph/0311129] [InSPIRE].

[13] T. Hambye, J. March-Russell and S.M. West, TeV scale resonant leptogenesis from supersymmetry breaking, JHEP 07 (2004) 070 [hep-ph/0403183] [INSPIRE].

[14] A. Abada, H. Aissaoui and M. Losada, A model for leptogenesis at the TeV scale, Nucl. Phys. B 728 (2005) 55 [hep-ph/0409343] [INSPIRE].

[15] N. Sahu and U.A. Yajnik, Gauged B-L symmetry and baryogenesis via leptogenesis at TeV scale, Phys. Rev. D 71 (2005) 023507 [hep-ph/0410075] [INSPIRE].

[16] E.J. Chun, TeV leptogenesis in Z-prime models and its collider probe, Phys. Rev. D 72 (2005) 095010 [hep-ph/0508050] [INSPIRE].

[17] Z.-z. Xing and S. Zhou, Tri-bimaximal Neutrino Mixing and Flavor-dependent Resonant Leptogenesis, Phys. Lett. B 653 (2007) 278 [hep-ph/0607302] [INSPIRE].

[18] S.M. West, Neutrino masses and TeV scale resonant leptogenesis from supersymmetry breaking, Mod. Phys. Lett. A 21 (2006) 1629 [inSPIRE].

[19] N. Sahu and U. Sarkar, Predictive model for dark matter, dark energy, neutrino masses and leptogenesis at the TeV scale, Phys. Rev. D 76 (2007) 045014 [hep-ph/0701062] [INSPIRE].

[20] M. Abbas and S. Khalil, Neutrino masses, mixing and leptogenesis in TeV scale B-L extension of the standard model, JHEP 04 (2008) 056 [arXiv:0707.0841] [InSPIRE].

[21] J.-M. Frere, T. Hambye and G. Vertongen, Is leptogenesis falsifiable at LHC?, JHEP 01 (2009) 051 [arXiv: 0806.0841] [INSPIRE].

[22] S. Blanchet, Z. Chacko, S.S. Granor and R.N. Mohapatra, Probing Resonant Leptogenesis at the LHC, Phys. Rev. D 82 (2010) 076008 [arXiv:0904.2174] [INSPIRE].

[23] M. Aoki and S. Kanemura, Probing the Majorana nature of TeV-scale radiative seesaw models at collider experiments, Phys. Lett. B 689 (2010) 28 [arXiv:1001.0092] [INSPIRE].

[24] Y.H. Ahn and C.-S. Chen, Non-zero $U_{e 3}$ and TeV-Leptogenesis through $A_{4}$ symmetry breaking, Phys. Rev. D 81 (2010) 105013 [arXiv:1001.2869] [INSPIRE].

[25] S. Blanchet, P.S.B. Dev and R.N. Mohapatra, Leptogenesis with TeV Scale Inverse Seesaw in SO(10), Phys. Rev. D 82 (2010) 115025 [arXiv: 1010.1471] [InSPIRE].

[26] S. Iso, N. Okada and Y. Orikasa, Resonant Leptogenesis in the Minimal B-L Extended Standard Model at TeV, Phys. Rev. D 83 (2011) 093011 [arXiv:1011.4769] [inSPIRE].

[27] A. Ibarra, E. Molinaro and S.T. Petcov, Low Energy Signatures of the TeV Scale See-Saw Mechanism, Phys. Rev. D 84 (2011) 013005 [arXiv:1103.6217] [INSPIRE]. 
[28] D. Suematsu, Thermal Leptogenesis in a TeV Scale Model for Neutrino Masses, Eur. Phys. J. C 72 (2012) 1951 [arXiv:1103.0857] [INSPIRE].

[29] S. Kanemura, T. Nabeshima and H. Sugiyama, TeV-Scale Seesaw with Loop-Induced Dirac Mass Term and Dark Matter from $\mathrm{U}(1)_{B-L}$ Gauge Symmetry Breaking, Phys. Rev. D 85 (2012) 033004 [arXiv:1111.0599] [INSPIRE].

[30] D. Choudhury, N. Mahajan, S. Patra and U. Sarkar, Radiative leptogenesis at the TeV scale, JCAP 04 (2012) 017 [arXiv: 1104.1851] [INSPIRE].

[31] W. Abdallah, D. Delepine and S. Khalil, TeV Scale Leptogenesis in B-L Model with Alternative Cosmologies, Phys. Lett. B 725 (2013) 361 [arXiv:1205.1503] [INSPIRE].

[32] N. Okada, Y. Orikasa and T. Yamada, Minimal Flavor Violation in the Minimal $\mathrm{U}(1)_{B-L}$ Model and Resonant Leptogenesis, Phys. Rev. D 86 (2012) 076003 [arXiv:1207.1510] [INSPIRE].

[33] S. Kanemura, T. Nabeshima and H. Sugiyama, Radiative type-I seesaw model with dark matter via $\mathrm{U}(1)_{B-L}$ gauge symmetry breaking at future linear colliders, Phys. Rev. D 87 (2013) 015009 [arXiv: 1207.7061] [INSPIRE].

[34] S. Davidson and M. Elmer, Similar Dark Matter and Baryon abundances with TeV-scale Leptogenesis, JHEP 10 (2012) 148 [arXiv:1208.0551] [INSPIRE].

[35] W. Chao and M.J. Ramsey-Musolf, Hidden from View: Neutrino Masses, Dark Matter and TeV-Scale Leptogenesis in a Neutrinophilic 2HDM, Phys. Rev. D 89 (2014) 033007 [arXiv: 1212.5709] [INSPIRE].

[36] S. Kashiwase and D. Suematsu, Leptogenesis and dark matter detection in a TeV scale neutrino mass model with inverted mass hierarchy, Eur. Phys. J. C 73 (2013) 2484 [arXiv: 1301.2087] [INSPIRE].

[37] C.S. Fong, M.C. Gonzalez-Garcia, E. Nardi and E. Peinado, New ways to TeV scale leptogenesis, JHEP 08 (2013) 104 [arXiv: 1305.6312] [INSPIRE].

[38] F.F. Deppisch, J. Harz and M. Hirsch, Falsifying High-Scale Leptogenesis at the LHC, Phys. Rev. Lett. 112 (2014) 221601 [arXiv:1312.4447] [INSPIRE].

[39] S. Iso, N. Okada and Y. Orikasa, Classically conformal B-L extended Standard Model, Phys. Lett. B 676 (2009) 81 [arXiv:0902 .4050] [INSPIRE].

[40] S. Iso, N. Okada and Y. Orikasa, The minimal B-L model naturally realized at TeV scale, Phys. Rev. D 80 (2009) 115007 [arXiv:0909.0128] [INSPIRE].

[41] S. Iso and Y. Orikasa, TeV Scale B-L model with a flat Higgs potential at the Planck scale: in view of the hierarchy problem, PTEP 2013 (2013) 023B08 [arXiv:1210.2848] [INSPIRE].

[42] M. Shaposhnikov and I. Tkachev, The $\nu M S M$, inflation and dark matter, Phys. Lett. B 639 (2006) 414 [hep-ph/0604236] [INSPIRE].

[43] T. Asaka, M. Laine and M. Shaposhnikov, Lightest sterile neutrino abundance within the nuMSM, JHEP 01 (2007) 091 [hep-ph/0612182] [INSPIRE].

[44] T. Asaka and H. Ishida, Flavour Mixing of Neutrinos and Baryon Asymmetry of the Universe, Phys. Lett. B 692 (2010) 105 [arXiv: 1004.5491] [INSPIRE].

[45] L. Canetti, M. Drewes, T. Frossard and M. Shaposhnikov, Dark Matter, Baryogenesis and Neutrino Oscillations from Right Handed Neutrinos, Phys. Rev. D 87 (2013) 093006 [arXiv: 1208.4607] [INSPIRE]. 
[46] G. Sigl and G. Raffelt, General kinetic description of relativistic mixed neutrinos, Nucl. Phys. B 406 (1993) 423 [INSPIRE].

[47] E.K. Akhmedov, V.A. Rubakov and A.Y. Smirnov, Baryogenesis via neutrino oscillations, Phys. Rev. Lett. 81 (1998) 1359 [hep-ph/9803255] [inSPIRE].

[48] T. Asaka and M. Shaposhnikov, The $\nu M S M$, dark matter and baryon asymmetry of the universe, Phys. Lett. B 620 (2005) 17 [hep-ph/0505013] [INSPIRE].

[49] L. Canetti and M. Shaposhnikov, Baryon Asymmetry of the Universe in the $\nu M S M, J C A P$ 09 (2010) 001 [arXiv: 1006.0133] [INSPIRE].

[50] J.-S. Gagnon and M. Shaposhnikov, Baryon Asymmetry of the Universe without Boltzmann or Kadanoff-Baym equations, Phys. Rev. D 83 (2011) 065021 [arXiv:1012.1126] [INSPIRE].

[51] T. Asaka, S. Eijima and H. Ishida, Kinetic Equations for Baryogenesis via Sterile Neutrino Oscillation, JCAP 02 (2012) 021 [arXiv:1112.5565] [INSPIRE].

[52] W. Buchmüller and S. Fredenhagen, Quantum mechanics of baryogenesis, Phys. Lett. B 483 (2000) 217 [hep-ph/0004145] [INSPIRE].

[53] A. Anisimov, W. Buchmüller, M. Drewes and S. Mendizabal, Leptogenesis from Quantum Interference in a Thermal Bath, Phys. Rev. Lett. 104 (2010) 121102 [arXiv:1001.3856] [INSPIRE].

[54] A. Anisimov, W. Buchmüller, M. Drewes and S. Mendizabal, Quantum Leptogenesis I, Annals Phys. 326 (2011) 1998 [Erratum ibid. 338 (2011) 376-377] [arXiv:1012.5821] [INSPIRE].

[55] M. Garny, A. Hohenegger, A. Kartavtsev and M. Lindner, Systematic approach to leptogenesis in nonequilibrium QFT: Vertex contribution to the CP-violating parameter, Phys. Rev. D 80 (2009) 125027 [arXiv: 0909.1559] [inSPIRE].

[56] M. Garny, A. Hohenegger, A. Kartavtsev and M. Lindner, Systematic approach to leptogenesis in nonequilibrium QFT: Self-energy contribution to the CP-violating parameter, Phys. Rev. D 81 (2010) 085027 [arXiv:0911.4122] [INSPIRE].

[57] M. Garny, A. Hohenegger and A. Kartavtsev, Medium corrections to the CP-violating parameter in leptogenesis, Phys. Rev. D 81 (2010) 085028 [arXiv:1002.0331] [INSPIRE].

[58] M. Garny, A. Hohenegger and A. Kartavtsev, Quantum corrections to leptogenesis from the gradient expansion, arXiv: 1005.5385 [INSPIRE].

[59] M. Beneke, B. Garbrecht, M. Herranen and P. Schwaller, Finite Number Density Corrections to Leptogenesis, Nucl. Phys. B 838 (2010) 1 [arXiv:1002.1326] [INSPIRE].

[60] B. Garbrecht, Leptogenesis: The Other Cuts, Nucl. Phys. B 847 (2011) 350 [arXiv: 1011.3122] [INSPIRE].

[61] B. Garbrecht, F. Glowna and M. Herranen, Right-Handed Neutrino Production at Finite Temperature: Radiative Corrections, Soft and Collinear Divergences, JHEP 04 (2013) 099 [arXiv: 1302.0743] [INSPIRE].

[62] B. Garbrecht, F. Glowna and P. Schwaller, Scattering Rates For Leptogenesis: Damping of Lepton Flavour Coherence and Production of Singlet Neutrinos, Nucl. Phys. B 877 (2013) 1 [arXiv: 1303.5498] [INSPIRE].

[63] T. Frossard, M. Garny, A. Hohenegger, A. Kartavtsev and D. Mitrouskas, Systematic approach to thermal leptogenesis, Phys. Rev. D 87 (2013) 085009 [arXiv:1211.2140] [INSPIRE]. 
[64] T. Frossard, A. Kartavtsev and D. Mitrouskas, Systematic approach to $\Delta L=1$ processes in thermal leptogenesis, Phys. Rev. D 87 (2013) 125006 [arXiv:1304.1719] [INSPIRE].

[65] A. De Simone and A. Riotto, Quantum Boltzmann Equations and Leptogenesis, JCAP 08 (2007) 002 [hep-ph/0703175] [INSPIRE].

[66] A. De Simone and A. Riotto, On Resonant Leptogenesis, JCAP 08 (2007) 013 [arXiv: 0705.2183] [INSPIRE].

[67] B. Garbrecht and M. Herranen, Effective Theory of Resonant Leptogenesis in the Closed-Time-Path Approach, Nucl. Phys. B 861 (2012) 17 [arXiv:1112.5954] [INSPIRE].

[68] A. Abada, S. Davidson, F.-X. Josse-Michaux, M. Losada and A. Riotto, Flavor issues in leptogenesis, JCAP 04 (2006) 004 [hep-ph/0601083] [INSPIRE].

[69] E. Nardi, Y. Nir, E. Roulet and J. Racker, The importance of flavor in leptogenesis, JHEP 01 (2006) 164 [hep-ph/0601084] [INSPIRE].

[70] V. Cirigliano, A. De Simone, G. Isidori, I. Masina and A. Riotto, Quantum Resonant Leptogenesis and Minimal Lepton Flavour Violation, JCAP 01 (2008) 004 [arXiv:0711.0778] [INSPIRE].

[71] M. Beneke, B. Garbrecht, C. Fidler, M. Herranen and P. Schwaller, Flavoured Leptogenesis in the CTP Formalism, Nucl. Phys. B 843 (2011) 177 [arXiv: 1007.4783] [INSPIRE].

[72] M. Drewes and B. Garbrecht, Leptogenesis from a GeV Seesaw without Mass Degeneracy, JHEP 03 (2013) 096 [arXiv: 1206.5537] [INSPIRE].

[73] M. Flanz, E.A. Paschos, U. Sarkar and J. Weiss, Baryogenesis through mixing of heavy Majorana neutrinos, Phys. Lett. B 389 (1996) 693 [hep-ph/9607310] [INSPIRE].

[74] A. Pilaftsis, Resonant CP-violation induced by particle mixing in transition amplitudes, Nucl. Phys. B 504 (1997) 61 [hep-ph/9702393] [INSPIRE].

[75] A. Pilaftsis, Heavy Majorana neutrinos and baryogenesis, Int. J. Mod. Phys. A 14 (1999) 1811 [hep-ph/9812256] [INSPIRE].

[76] W. Buchmüller and M. Plümacher, CP asymmetry in Majorana neutrino decays, Phys. Lett. B 431 (1998) 354 [hep-ph/9710460] [INSPIRE].

[77] A. Anisimov, A. Broncano and M. Plümacher, The CP-asymmetry in resonant leptogenesis, Nucl. Phys. B 737 (2006) 176 [hep-ph/0511248] [INSPIRE].

[78] R. Rangarajan and H. Mishra, Leptogenesis with heavy Majorana neutrinos revisited, Phys. Rev. D 61 (2000) 043509 [hep-ph/9908417] [INSPIRE].

[79] M. Garny, A. Kartavtsev and A. Hohenegger, Leptogenesis from first principles in the resonant regime, Annals Phys. 328 (2013) 26 [arXiv:1112.6428] [INSPIRE].

[80] P.S.B. Dev, P. Millington, A. Pilaftsis and D. Teresi, Flavour covariant transport equations: an application to resonant leptogenesis, Nucl. Phys. B 886 (2014) 569 [arXiv:1404.1003] [INSPIRE].

[81] V.G. Morozov and G. Ropke, Extended quasiparticle approximation for relativistic electrons in plasmas, Condens. Matter Phys. 9 (2006) 473. 